\title{
Frequency Transition from Diffusion to Capacitive Response in the Blocked-Diffusion Warburg Impedance for EIS Analysis in Modern Batteries
}

To cite this article before publication: Samuel Cruz-Manzo et al $2020 \mathrm{~J}$. Electrochem. Soc. in press https://doi.org/10.1149/1945-7111/abbfdc

\section{Manuscript version: Accepted Manuscript}

Accepted Manuscript is "the version of the article accepted for publication including all changes made as a result of the peer review process, and which may also include the addition to the article by IOP Publishing of a header, an article ID, a cover sheet and/or an 'Accepted Manuscript' watermark, but excluding any other editing, typesetting or other changes made by IOP Publishing and/or its licensors"

This Accepted Manuscript is $\odot 2020$ The Author(s). Published by IOP Publishing Ltd..

This article can be copied and redistributed on non commercial subject and institutional repositories.

Although reasonable endeavours have been taken to obtain all necessary permissions from third parties to include their copyrighted content within this article, their full citation and copyright line may not be present in this Accepted Manuscript version. Before using any content from this article, please refer to the Version of Record on IOPscience once published for full citation and copyright details, as permissions will likely be required. All third party content is fully copyright protected, unless specifically stated otherwise in the figure caption in the Version of Record.

View the article online for updates and enhancements. 
Frequency Transition from Diffusion to Capacitive Response in the Blocked-Diffusion Warburg Impedance for EIS

\section{Analysis in Modern Batteries}

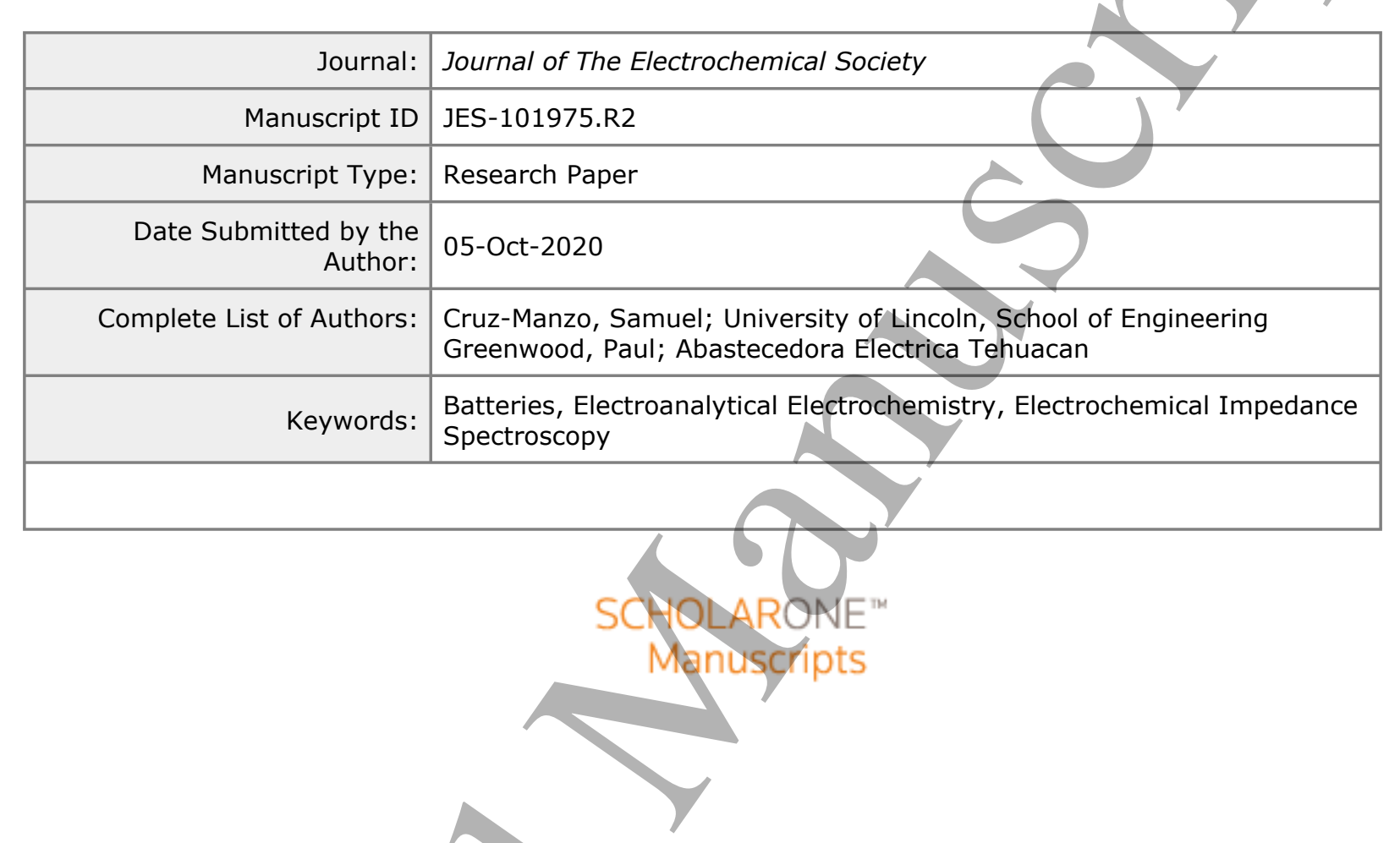




\title{
Frequency Transition from Diffusion to Capacitive Response in the Blocked-Diffusion Warburg Impedance for EIS Analysis in Modern
} Batteries

\author{
Samuel Cruz-Manzo ${ }^{1, z}$ and Paul Greenwood ${ }^{2}$ \\ ${ }^{1}$ School of Engineering, University of Lincoln, Lincolnshire LN6 7TS, United Kingdom \\ ${ }^{2}$ Abastecedora Electrica Tehuacan, Engineering Division, Tehuacan, Puebla 75700, Mexico \\ zE-mail: s.cruz-manzo@ hotmail.com
}

\begin{abstract}
The use of the Blocked-diffusion Warburg (BDW) impedance within electrochemical impedance spectroscopy (EIS) measurements can unveil/diffusion properties of the electroactive material of modern batteries at different states-of-charge. The impedance response of the BDW comprises a diffusion response of charge carriers through a shortdiffusion distance (e.g. the solid-phase in electroactive material of battery electrodes) and a capacitive response due to accumulation of charge carriers in a blocked-interface (e.g. impermeable current collector of a thin film electrode). This study has developed a mathematical expression based on the Newton-Raphson iteration method to calculate the frequency and time constant during the transition from diffusion to capacitive response in the BDW impedance. The mathematical procedure to calculate the frequency during the diffusioncapacitive transition response in the BDW has been written in a script in Matlab® software and is applied to BDW impedance responses reported in previous studies and extracted from EIS measurements in Li-ion and NiMH batteries. This study demonstrates that the time constant during the transition from diffusion to capacitive response in the BDW differs from the characteristic time constant cormmonly represented in the BDW mathematical expression. The characteristic time constant represented in the BDW mathematical expression is related to the rate of accumulation of charge carriers in the blocked-interface of the electrode. On the other hand, the time constant during the transition from diffusion to capacitance responses in the BDW impedance can be related to diffusion properties in solid-phase particles with heterogeneous size distribution in the electroactive material of modern battery electrodes.
\end{abstract}




\section{Introduction}

Electrochemical impedance spectroscopy (EIS) is a nonintrusive experimental technique which has been broadly applied to characterise phenomenological processes in electrochemical systems. The use of equivalent electrical circuits (EECs) within the EIS technique has been the most accepted method to estimate electrochemical and diffusion mechanisms in electrochemical systems. EECs with Warburg impedances have been considered to characterise diffusion mechanisms in EIS-battery measurements carried out at different stateof-charge, (SoC) $[1,2,3,4,5,6,7,8]$. The mathematical expression of the Warburg impedance has been derived from the Second's Fick Law in the frequency domain using Laplace transformation. The Warburg impedance can be classified as semi-infinite diffusion Warburg (SDW), permeable finite-length Warburg (FLW), and blocked-diffusion Warburg (BDW). This classification results from the derivation of Fick's Second Law of Diffusion with different boundary conditions, and the boundary conditions will be defined from the diffusion process of the electrochemical system studied. The SDW impedance considers diffusion of a particle in semi-infinite space [9]. The impedance response of the SDW can be represented by a line with a slope of 1 (or $45^{\circ}$ with respect to the real axis) in the Nyquist plot. The FLW has been commonly applied to characterise oxygen transport limitations in EIS measurements of polymer electrolyte fuel cells (PEFCs) [10,11]. The FLW impedance response can be simulated by an infinite series combination of parallel resistor-capacitor circuits [12]. The BDW impedance is attributed to impermeable or blocking boundary conditions. Blocked-diffusion mechanisms can be manifested when charge carriers cannot penetrate through the back of the electrode, but they accumulate at the metal/electrode interface resulting in a capacitive effect [13]. The BDW impedance response is represented by a transition from a $45^{\circ}$ straight-line at high frequencies to a vertical line in the Nyquist plot at low frequencies. The $45^{\circ}$ straight-line response is attributed to diffusion of charge carriers across the solid-phase of the electroactive material and the vertical line response is attributed to accumulation of charge carriers in a blocked-interface. The active material in a battery electrode can be comprised of solid-particles with different geometry (e.g. planar, spherical, cylindrical) and heterogenous size distribution. The blocked-interface can be related to the current collector in a battery electrode. Fig. 1 shows the interface between the active material comprised of spherical solid particles and current collector in a battery electrode where $\delta_{s}$ can be related to the actual geometry of the solid particle and $\delta$ is the total diffusion distance of ions moving from the solid-phase particles to the blocked-interface (e.g. current collector) in the electrode. The blocked-diffusion 
mechanisms can also be manifested when carrying out EIS measurements in batteries at opencircuit voltage (OCV). A deviation (frequency dispersion) of the frequency response from the ideal frequency response of the impedance diffusion of a battery can be manifested. Frequency dispersion in the impedance diffusion of an electrode has been attributed to non-homogenous charge distribution in the electrode due to roughness of the material [14] and heterogenous particle size [15] in the active material. The theory of anomalous diffusion, diffusion and trapping impedance in intercalation materials have been considered to predict frequency dispersion in the impedance diffusion of electroactive materials [16,17]. Under these conditions a BDW impedance with frequency dispersion can be defined [18]. Some studies have been focused on the interpretation and estimation of the different time constants associated with the Warburg impedance. Barbero [19] reported a theoretical interpretation of the FLW impedance based on the Poisson-Nernst-Planck model. Nguyen and Breitkopf [20] develop an algorithm to calculate the different time constants associated with the impedance response generated from a Randles circuit containing a FLW impedance. T.M.W.J. Bandara and B.-E. Mellander [21] estimated the time constants represented in the impedance response of a Randles circuit connected in series with a capacitor representing a blocking electrode. The authors reported a mathematical expression for the frequency during the transition from a semicircle to a straightline feature at low frequencies in the Nyquist plot. The frequency associated to the transition from diffusion to capacitive response in the BDW has been estimated through a graphical interpretation of the impedance response on the Nyquist plot [16,22]. However, this graphical interpretation cannot be carried out if the BDW impedance response is overlapped with other frequency response mechanisms on the overall EIS response of the electrode [15]. In this study a mathematical expression to estimate the frequency and time constant during the transition from diffusion response to capacitive response in the BDW impedance has been developed. The methodology developed in this study can be extended and applied to battery impedance models comprising different BDW configuration and representing different particle geometry in the electrode as the diffusion-capacitive transition response at low frequencies is represented in EIS measurements carried out in electrodes with different particle geometry [15]. This study could assist the understanding of diffusion mechanisms in modern batteries and could also provide an insight into diffusion properties in the solid-phase of the active material of modern battery electrodes using EIS. 


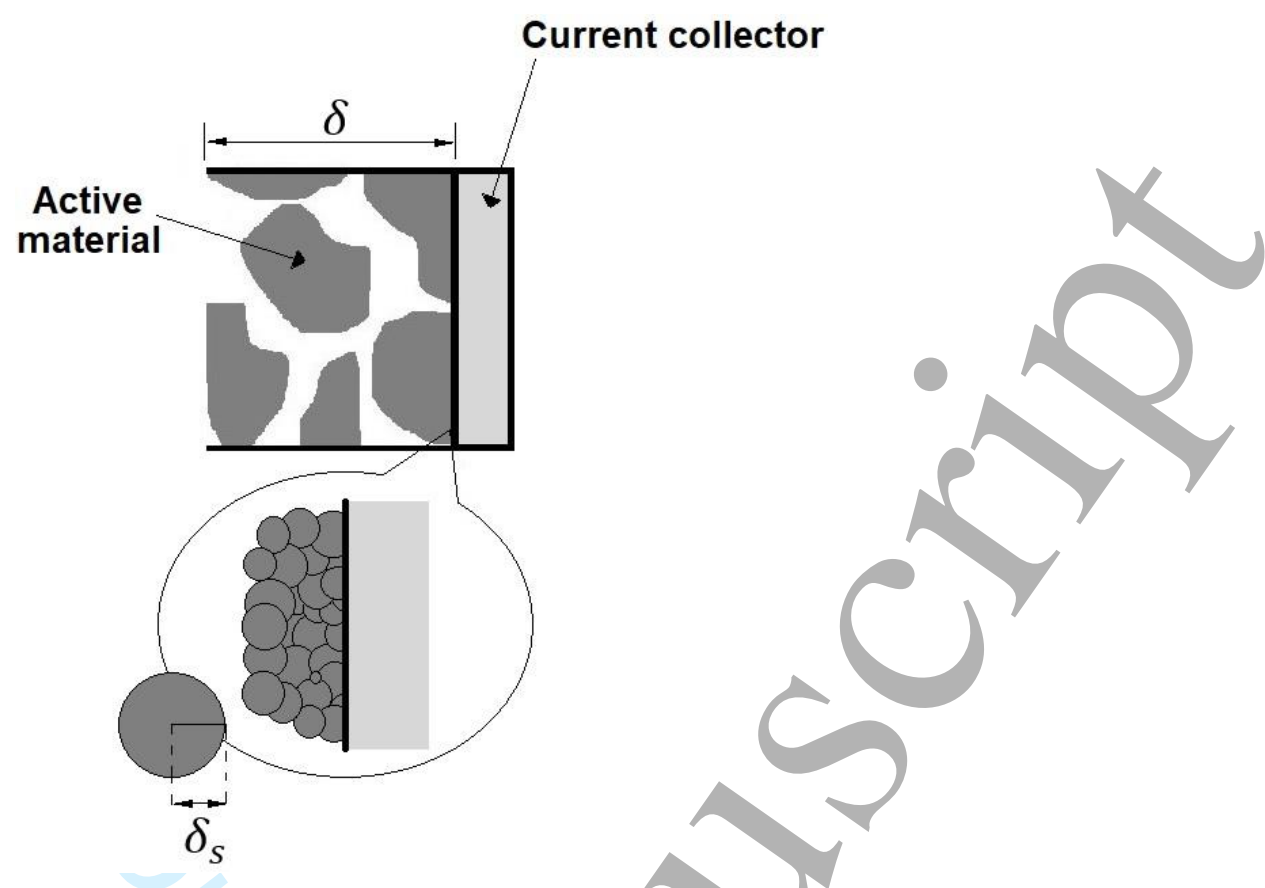

Figure 1. Active material /blocked interface (current collector) in electrode.

\section{Representation of Warburg impedance response}

Figure 2 shows the impedance response of the FLW and BDW. The impedance response of the BDW with frequency dispersion is shown in Fig. 2 as well. A transition from a $45^{\circ}$ straightline to a vertical line in the BDW impedance response is observed in Fig. 2a. A transition from 45 degree in phase angle to 90 degree in phase angle of the BDW impedance response is shown in Fig. 2b. The 45 degree in the phase angle of the BDW impedance shown in Fig. $2 \mathrm{~b}$ is attributed to diffusion of charge carriers (e.g. ions) through a short-diffusion length in the solidphase of the electroactive material in the electrode. The 90 degree in the phase angle of the BDW impedance shown in Fig. $2 b$ is attributed to accumulation of charge carriers in the blocked-interface of an electrode (e.g. impermeable current collector of a thin film electrode or the reflective centre of a nanoparticle at accessible low frequencies [15]). The transition from the $45^{\circ}$ straight-line to the vertical line in the BDW shown in Fig. $2 \mathrm{a}$ is attributed to the diffusion of charge carriers in a short-diffusion length of the solid-phase particles which thereafter the charge carriers are accumulated in the blocked-interface of the electrode behaving similar to the electric charge in a capacitor and resulting in a vertical line in the impedance response at low frequencies. As previously mentioned different factors such as geometry $[14,15]$, anomalous diffusion [16] yield a nonhomogenous accumulation of charge (frequency dispersion) in the blocked-interface of the electrode. A nonhomogeneous accumulation of charge carriers (frequency dispersion) in the blocked-interface yields a vertical 
line with a slope in the impedance diffusion response (BDW with frequency dispersion) as shown in Fig. 2a. Oldenburger et al. [23] demonstrated that the change of slope in the vertical line of the BDW at low frequencies can also be attributed to hysteresis effect during EIS measurements in Li-ion cells.

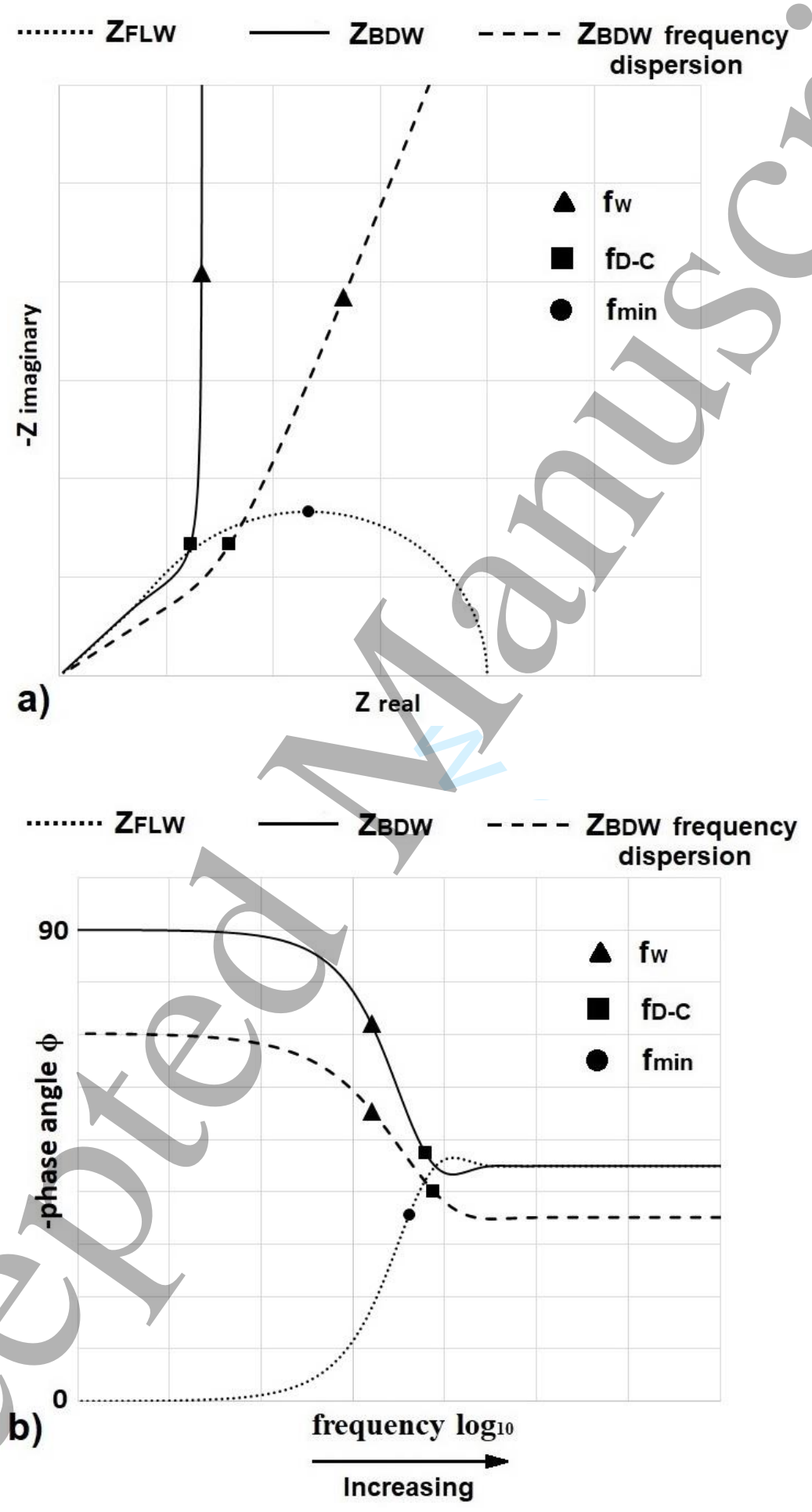

Figure 2. Impedance responses for finite-length Warburg (FLW) and Blocked-diffusion Warburg (BDW), a) Nyquist plot, b) phase angle 
The characteristic time constant for the diffusion process is commonly considered in the equations that represent the FLW [10,24] and BDW impedances [25,26]. The characteristic frequency $f_{W}$ calculated from the characteristic time constant and represented in the phase angle of the BDW impedance shown in Fig. $2 \mathrm{~b}$ considers that around $60 \%$ of the charge carriers have accumulated in the blocked-interface before reaching a full accumulation at 90 degrees in phase angle and at lower frequencies. The time constant of a first order system such as the Randles circuit (resistor-capacitor configuration) represents the transient output response which has reached $63.2 \%$ of its total change [27]. The diffusion and accumulation of charge carriers from the solid-phase to the blocked-interface can be represented in the phase angle of the BDW impedance as shown in Fig. 3. The characteristic frequency $f_{W}$ shown in Fig. 3 is lower than the frequency $f_{D-C}$ related to the diffusion-capacitive transition response (kink of the BDW impedance response shown in Fig. 2a). The estimation of the time constant $T_{D-C}$ during the transition from diffusion response to the capacitive response in the BDW impedance shown in Figs. 2 and 3 could provide relevant information about diffusion properties in the solid-phase of particles of modern battery electrodes. It is possible to define a diffusion coefficient of charge carriers in the solid-phase of active material related to the frequency transition $f_{D-C}$ [22] which is different from the diffusion coefficient related to the characteristic frequency $f_{W}$ in the BDW impedance.

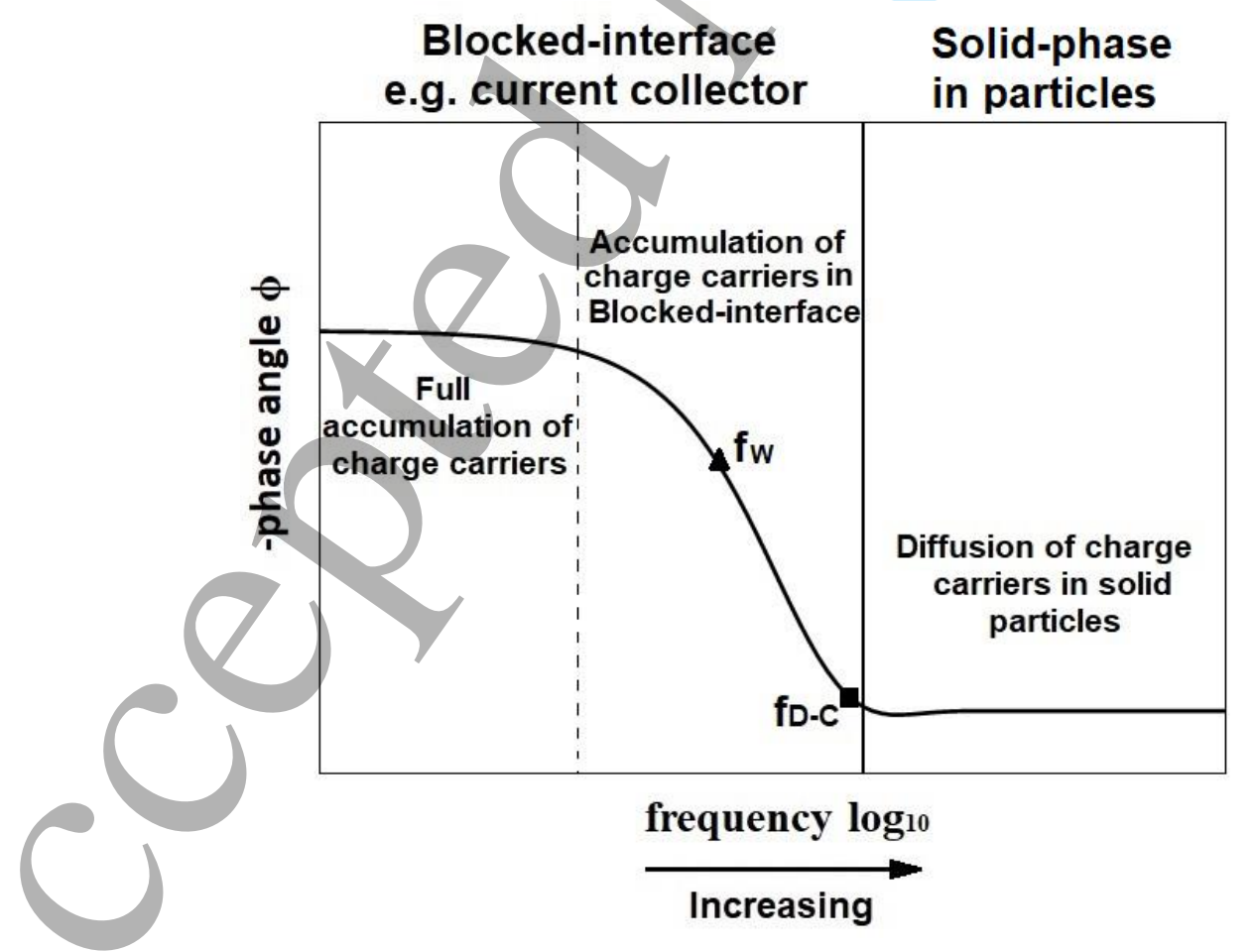

Figure 3. Representation of diffusion mechanisms on the phase angle of the BDW impedance 
In the literature, a mathematical equation has been derived [28] to calculate the frequency $f_{\min }$ at which the imaginary component $\mathrm{Z}_{\mathrm{im}}$ of the FLW impedance response reaches at minimum value in the Nyquist plot as shown in Fig. 2a. This study carries out a step by step mathematical calculation of $f_{\min }$ in the FLW impedance response using the Newton-Raphson iteration method. The mathematical procedure to calculate $f_{\min }$ in the FLW impedance response will be considered a baseline for the development of a mathematical expression to estimate the frequency $\mathrm{f}_{\mathrm{D}-\mathrm{C}}$ during the transition from diffusion to capacitive responses in the BDW impedance. The mathematical procedure to estimate the frequency $f_{D-C}$ from the BDW impedance is based on the Newton method with a fixed-point iteration and has been written in a script in Matlab® software.

It is possible to estimate the frequency $f_{D-C}$ through a graphical interpretation of the BDW impedance response in the Nyquist plot (kink of the curve as shown in Fig. 2a) [22,29]. When the slope of the straight-line at low frequencies decreases in the BDW with frequency dispersion, it becomes more complicated to visually distinguish between the diffusion and capacitive responses in the BDW impedance response. In addition, the BDW impedance represented in the EIS response of batteries may be overlapped with other electrochemical mechanisms during the redox reaction making difficult the estimation of the frequency $f_{D-C}$ through a visual inspection of the Nyquist plot. Song et al. [15] reported that EIS measurements carried out in $\mathrm{Li}$-ion batteries at low $\mathrm{Li}^{+}$concentration results in an overlapping effect between the semicircle at high frequencies related to the charge transfer reaction and the BDW response at low frequencies represented in the Nyquist plot.

In a previous study [18], an EEC comprised of a BDW considering frequency dispersion was fitted to EIS measurements carried out in a NiMH battery at different SoC. The reported values of the parameters from the BDW mathematical expression are considered to simulate the impedance diffusion response of the BDW at different SoC. The developed mathematical expression to calculate the frequency transition $f_{D-C}$ from diffusion to capacitor response is considered within the BDW impedance response extracted from the EIS measurements in the NiMH battery reported in the previous study [18]. It is possible to gain an insight into diffusion mechanisms in the solid-phase electroactive material of the electrode during the discharge of the NiMH battery. 


\section{Analysis using the Finite-length Warburg impedance}

The equation that represents the FLW impedance is expressed as follows [24]:

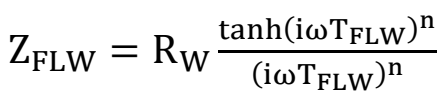

where $\mathrm{n}=0.5, \omega=2 \pi \mathrm{f}$ is the angular frequency and $i$ is the imaginary component of a complex number.

$\mathrm{R}_{\mathrm{W}}=\frac{\mathrm{RT} \delta}{\mathrm{z}^{2} \mathrm{~F}^{2} \mathrm{c}_{\mathrm{O}}^{*} \mathrm{D}}$

is defined as resistance for the diffusion process of chemical species and

$\mathrm{T}_{\mathrm{FLW}}=\frac{\delta^{2}}{\mathrm{D}}$

defined as the time constant to diffuse chemical species through a finite-length medium.

The impedance response generated by Eq. 1 has been depicted in Fig. 2. Eq. 1 can be separated into real and imaginary components by considering trigonometric relations [12,20]. Eq. 1 can be expressed as:

$\mathrm{Z}_{\mathrm{FLW}}=\mathrm{R}_{\mathrm{W}} \frac{\tanh (\alpha+\mathrm{i} \beta)}{\alpha+\mathrm{i} \beta}$

where

$\alpha=\mathrm{s}^{\mathrm{n}} \cos (\mathrm{n} \pi / 2)$

and

$\beta=s^{\mathrm{n}} \sin (\mathrm{n} \pi / 2)$, 
Eqs. 5 and 6 have been estimated considering the following relations: $\mathrm{s}=\omega \mathrm{T}_{\mathrm{FLW}}$, $(\mathrm{is})^{\mathrm{n}}=$ $s^{n} e^{n i \pi / 2}$, and $e^{n i \pi / 2}=\cos (n \pi / 2)+i \sin (n \pi / 2)$.

Multiplying Eq. 4 by the conjugate of the denominator and considering trigonometric identity for tanh yields:

$\mathrm{Z}_{\mathrm{FLW}}=\frac{\mathrm{R}_{\mathrm{W}}(\alpha-\mathrm{i} \beta)}{\alpha^{2}+\beta^{2}} \frac{\sinh (\alpha+\mathrm{i} \beta)}{\cosh (\alpha+\mathrm{i} \beta)}$

Considering trigonometric identities in Eq. 7 e.g. $\sinh (\alpha+i \beta)=\frac{\mathrm{e}^{\alpha+\mathrm{i} \beta}-\mathrm{e}^{-\alpha-\mathrm{i} \beta}}{2}$ yields:

$Z_{F L W}=\frac{R_{W}(\alpha-i \beta)}{\alpha^{2}+\beta^{2}} \frac{e^{\alpha} e^{i \beta}-e^{-\alpha} e^{-i \beta}}{e^{\alpha} e^{i \beta}+e^{-\alpha} e^{-i \beta}}$

Considering Euler formula $\mathrm{e}^{ \pm \mathrm{i} \beta}=\cos \beta \pm \mathrm{i} \sin \beta$ and trigonometric identities, e.g. $\cosh (\alpha+\mathrm{i} \beta)=\frac{\mathrm{e}^{\alpha+\mathrm{i} \beta}+\mathrm{e}^{-\alpha-\mathrm{i} \beta}}{2}$ and arranging Eq. 8 yields:

$\mathrm{Z}_{\mathrm{FLW}}=\frac{\mathrm{R}_{\mathrm{W}}(\alpha-\mathrm{i} \beta)}{\alpha^{2}+\beta^{2}} \frac{\cos \beta \sinh \alpha+\mathrm{i} \sin \beta \cosh \alpha}{\cos \beta \cosh \alpha+\mathrm{i} \sin \beta \sinh \alpha}$

Eq. 9 can be defined as:

$\mathrm{Z}_{\mathrm{FLW}}=\frac{\mathrm{R}_{\mathrm{W}}(\alpha-\mathrm{i} \beta)}{\alpha^{2}+\beta^{2}} \frac{\mathrm{ad}+\mathrm{ibc}}{\mathrm{ac}+\mathrm{ibd}}$

where $\mathrm{a}=\cos \beta, \mathrm{b}=\sin \beta, \mathrm{c}=\cosh \alpha$, and $\mathrm{d}=\sinh \alpha$. Multiplying Eq. 10 by the conjugate of the denominator yields:

$Z_{\mathrm{FLW}}=\frac{\mathrm{R}_{\mathrm{W}}(\alpha-i \beta)}{\alpha^{2}+\beta^{2}} \frac{c \mathrm{~d}\left(\mathrm{a}^{2}+\mathrm{b}^{2}\right)+\mathrm{iab}\left(\mathrm{c}^{2}-\mathrm{d}^{2}\right)}{\mathrm{a}^{2} \mathrm{c}^{2}+\mathrm{b}^{2} \mathrm{~d}^{2}}$

Substituting a, b, c, and d in Eq. 11 as previously defined in Eq. 10 yields:

$Z_{\mathrm{FLW}}=\frac{\mathrm{R}_{\mathrm{W}}(\alpha-i \beta)}{\alpha^{2}+\beta^{2}} \frac{\cosh \alpha \sinh \alpha\left(\cos ^{2} \beta+\sin ^{2} \beta\right)+\mathrm{i} \cos \beta \sin \beta\left(\cosh ^{2} \alpha-\sinh ^{2} \alpha\right)}{\cos ^{2} \beta \cosh ^{2} \alpha+\sin ^{2} \beta \sinh ^{2} \alpha}$ 
Considering trigonometric identities e.g. $\cos ^{2} \beta+\sin ^{2} \beta=1, \quad \cosh ^{2} \alpha-\sinh ^{2} \alpha=1$, $2 \sinh \alpha \cosh \alpha=\sinh 2 \alpha, \cosh ^{2} \alpha=(\cosh 2 \alpha+1) / 2$, and arranging Eq. 12 yields:

$\mathrm{Z}_{\mathrm{FLW}}=\frac{\mathrm{R}_{\mathrm{W}}(\alpha-\mathrm{i} \beta)}{\alpha^{2}+\beta^{2}} \frac{\sinh 2 \alpha+\mathrm{i} \sin 2 \beta}{\cosh 2 \alpha+\cos 2 \beta}$

Eq. 13 can be separated into real and imaginary components $\mathrm{Z}_{\mathrm{FLW}}=\mathrm{Z}_{\mathrm{FLW} \_\mathrm{Re}}+\mathrm{iZ}_{\mathrm{FLW} \_\mathrm{Im}}$ as such:

$\mathrm{Z}_{\mathrm{FLW}}=\overbrace{\frac{\mathrm{R}_{\mathrm{W}}}{\alpha^{2}+\beta^{2}} \frac{\alpha \sinh 2 \alpha+\beta \sin 2 \beta}{\cosh 2 \alpha+\cos 2 \beta}}^{\mathrm{Z}_{\mathrm{FLW} \text { Re }}}+\mathrm{i} \overbrace{\frac{\mathrm{R}_{\mathrm{W}}}{\alpha^{2}+\beta^{2}} \frac{\alpha \sin 2 \beta-\beta \sinh 2 \alpha}{\cosh 2 \alpha+\cos 2 \beta}}^{\mathrm{Z}_{\mathrm{FLW} \_\mathrm{Im}}}$

Substituting the parameters $\alpha$ and $\beta$ defined in Eqs. 5 and 6 into the second term on the righthand side of Eq. 14 that represents the imaginary component yields:

$\mathrm{Z}_{\mathrm{FLW} \_\mathrm{Im}}=\frac{\mathrm{R}_{\mathrm{W}}}{\mathrm{s}^{\mathrm{n}}} \frac{\cos (\mathrm{n} \pi / 2) \sin \left[2 \mathrm{~s}^{\mathrm{n}} \sin (\mathrm{n} \pi / 2)\right\rfloor-\sin (\mathrm{n} \pi / 2) \sinh \left[2 \mathrm{~s}^{\mathrm{n}} \cos (\mathrm{n} \pi / 2)\right\rfloor}{\cos \left[2 \mathrm{~s}^{\mathrm{n}} \sin (\mathrm{n} \pi / 2)\right\rfloor+\cosh \left[2 \mathrm{~s}^{\mathrm{n}} \cos (\mathrm{n} \pi / 2)\right\rfloor}$

If $n=0.5$ in Eq. 15 and considering that $\cos (\pi / 4)=\sin (\pi / 4)=\sqrt{2} / 2$, it is possible to represent the imaginary component of the FLW impedance, as such:

$\mathrm{Z}_{\mathrm{FLW} \_\mathrm{Im}}=\frac{\mathrm{R}_{\mathrm{W}}}{\sqrt{2 \mathrm{~s}}} \frac{\sin \sqrt{2 \mathrm{~s}}-\sinh \sqrt{2 \mathrm{~s}}}{\cos \sqrt{2 \mathrm{~s}}+\cosh \sqrt{2 \mathrm{~s}}}$

Note that Eqs. 15 and 16 do not include the imaginary unit $i$, but the numbers generated from Eqs. 15 and 16 represent the imaginary numbers of the impedance $\mathrm{Z}_{\mathrm{FLW}}$ represented in Eq. 14 .

Fig. 4 shows the imaginary components of the FLW generated from Eq. 16 at different frequencies $\omega$ considering $\mathrm{s}=\omega \mathrm{T}_{\mathrm{FLW}}$ and the local minimum value represented through the zero slope. The minimum value of a function $\mathrm{F}(\mathrm{x})$ can be calculated through the estimation of its roots when the derivative of the function is equal to zero as $\operatorname{such} \mathrm{F}^{\prime}(\mathrm{x})=0$. 


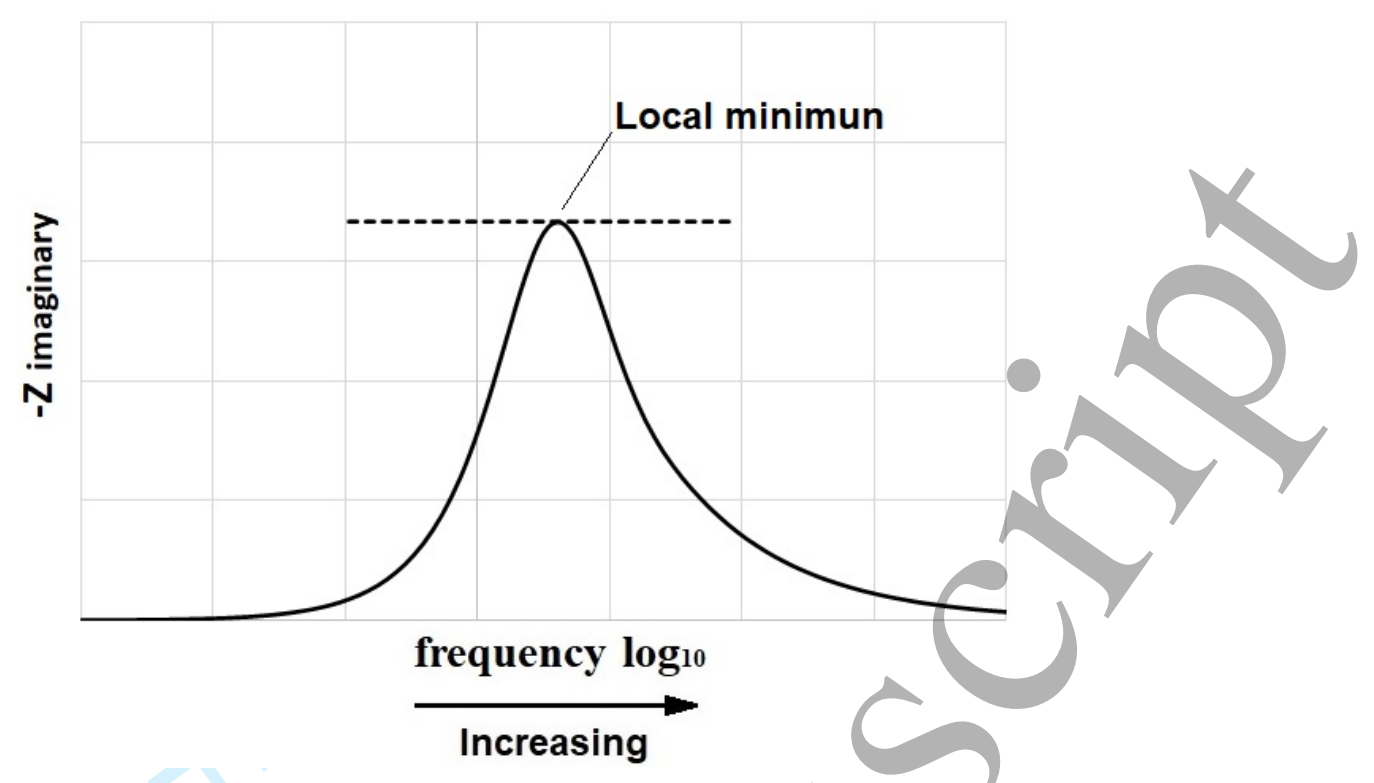

Figure 4. Imaginary component as a function of the frequency in FLW (Eq. 16).

\subsection{Newton-Raphson iteration method}

The value where imaginary components of the FLW generated through Eq. 16 at different frequencies reaches a minimum value (minimum of the function) can be estimated through the Newton-Raphson method. The iterative process of the Newton-Raphson method can be expressed as follows:

$x_{j+1}=x_{j}-\frac{F\left(x_{j}\right)}{F^{\prime}\left(x_{j}\right)}$

where $\mathrm{j}=0,1,2, \ldots \mathrm{k}$ is the iterative sequence of the root $x$ to be found on the function $\mathrm{F}(\mathrm{x})$ with the condition $\mathrm{F}(\mathrm{x})=0$. One of the disadvantages of the iteration process represented in Eq. 17 is that if the converged solution for $x$ corresponds to a zero slope in the function when $\mathrm{F}^{\prime}(\mathrm{x})=0$, then the iterative sequence represented in Eq. 17 will never converge on a solution. This is the case for the function $\mathrm{Z}_{\mathrm{FLW} \_ \text {Im }}(\mathrm{s})$ represented in Eq. 16 and shown in Fig. 4 in which it is required to find $s$ when the derivative of the function is equal to zero $Z_{F L W \_I m}{ }^{\prime}(s)=0$.

The local minimum of a function when $\mathrm{F}^{\prime}(\mathrm{x})=0$ can be estimated through the Newton method considered as an optimization algorithm. The local minimum value of the function $Z_{\text {FLW_Im }}(s)$ considering the condition $\mathrm{Z}_{\mathrm{FLW} \_\mathrm{Im}}{ }^{\prime}(\mathrm{s})=0$ can be estimated through a modification of the Newton-Raphson iteration method as such: 
$s_{j+1}=s_{j}-\frac{Z_{F L W_{-} I m}{ }^{\prime}\left(s_{j}\right)}{Z_{F L W_{-} I m}{ }^{\prime \prime}\left(s_{j}\right)}$

$\mathrm{j}=0,1,2, \ldots \mathrm{k}$ is the iterative sequence for a converged solution $s_{k}$ to be found and $\mathrm{Z}_{\mathrm{FLW}} \mathrm{Im}$ and $\mathrm{Z}_{\mathrm{FLW} \_\mathrm{Im}}{ }^{\prime}$ are the first and second derivative of Eq. 16 with respect to s. Nowadays, the use of computational software allows the calculation of differential equations and the implementation of iterative processes. For instance, Matlab® is a programming language which allows symbolic programming and computing. It also allows to obtain the derivative of symbolic functions using defined syntaxes, e.g. the derivative of a symbolic function can be obtained with the syntaxis $Y=\operatorname{diff}(X)$. An initial guess $\mathrm{s}_{0}$ during the iteration process of Eq. 18 has to be defined, the number of iterations $s_{0}, s_{1}, s_{2}, \ldots . s_{\mathrm{k}}$ will depend on the proximity of the initial guest $\mathrm{s}_{0}$ to the converged solution for $\mathrm{Z}_{\mathrm{FLW} \_\mathrm{Im}}{ }^{\prime}(\mathrm{s})=0$.

\subsection{Frequency $f_{\min }$ at the minimum imaginary component of FLW impedance}

Malevich et al. [10] fitted an EEC comprising a FLW component within EIS measurements carried out in a PEFC. Parameters of the FLW reported by Malevich et al. [10] such as $\mathrm{R}_{\mathrm{W}}=$ 0.09 and $\mathrm{T}_{\mathrm{FLW}}=0.172$ have been considered to demonstrate the calculation of the frequency at the minimum imaginary component $Z_{\mathrm{im}}$ of the FLW impedance through the iteration process represented in Eq. 18. The iteration process represented in Eq. 18 does not require the parameter $\mathrm{T}_{\mathrm{FLW}}$ as this is contained in the nondimensional parameter $\mathrm{s}=\omega \mathrm{T}_{\mathrm{FLW}}$ in Eq. 16.

Table I shows the results of the iteration process of Eq. 18 and considering an initial value $s_{0}=$ 1. It also demonstrates that the solution converged with a value of $s_{k}=2.54$. The iteration process shown in Table I was calculated using a script written in Matlab® software as shown in Appendix A1. The converged solution $s_{k}=2.54$ will be achieved despite any value of $R_{W}$ in Eq. 16, as the parameter $R_{W}$ increases or decreases the magnitude of the bell-shaped curve shown in Fig. 4 without changing its shape. 


\begin{tabular}{|c|c|c|c|}
\hline $\mathrm{j}$ & $\mathrm{s}_{\mathrm{j}}$ & $\mathrm{s}_{\mathrm{j}+1}$ & error \% \\
\hline 0 & 1 & 2.06895 & 18.566 \\
\hline 1 & 2.06895 & 2.43791 & 4.044 \\
\hline 2 & 2.43791 & 2.53449 & 0.242 \\
\hline 3 & 2.53449 & 2.54062 & $9.269 \mathrm{e}-04$ \\
\hline 4 & 2.54062 & $\mathbf{2 . 5 4 0 6 4}$ & $1.363 \mathrm{e}-08$ \\
\hline
\end{tabular}

Table I. Iteration process considering the initial condition $s_{0}=1$ in Eq. 18 .

Based on the converged solution for $s_{k}=2.54$ expressed in Table $\mathrm{I}$ and considering $\mathrm{s}=$ $\omega \mathrm{T}_{\mathrm{FLW}}$, it is possible to define an equation to calculate the frequency $f_{\min }$ at which the imaginary component $Z_{\mathrm{im}}$ of the FLW impedance response reaches a minimum value, as such:

$\mathrm{f}_{\min }=\frac{2.54}{2 \pi \mathrm{T}_{\mathrm{FLW}}}$

Eq. 19 has been reported in the literature with a pre-factor of 2.53 instead [30,31]. Eq. 19 expresses that the time constant where the imaginary component $Z_{\text {im }}$ of the FLW reaches a minimum value $\mathrm{T}_{\min }=1 /\left(2 \pi \mathrm{f}_{\min }\right)$ is a factor 2.54 smaller than the characteristic time constant $\mathrm{T}_{\mathrm{FLW}}$ represented in the FLW equation (Eq. 1).

Fig. 5a shows the impedance spectrum of the FLW generated from Eq. 1 and the parameters $\mathrm{R}_{\mathrm{W}}=0.09$ and $\mathrm{T}_{\mathrm{FLW}}=0.172$ reported in the literature [10] for a range of frequencies $f=$ $10 \mathrm{kHz}-0.001 \mathrm{~Hz}$. Fig. $5 \mathrm{~b}$ shows the imaginary components $\mathrm{Z}_{\mathrm{im}}$ of the FLW impedance response as a function of frequency and calculated through Eq. 16. The frequency $f_{\min }$ at which the imaginary components $\mathrm{Z}_{\mathrm{im}}$ reached a minimum value and calculated through Eq. 19 was evaluated to be $2.35 \mathrm{~Hz}$. 


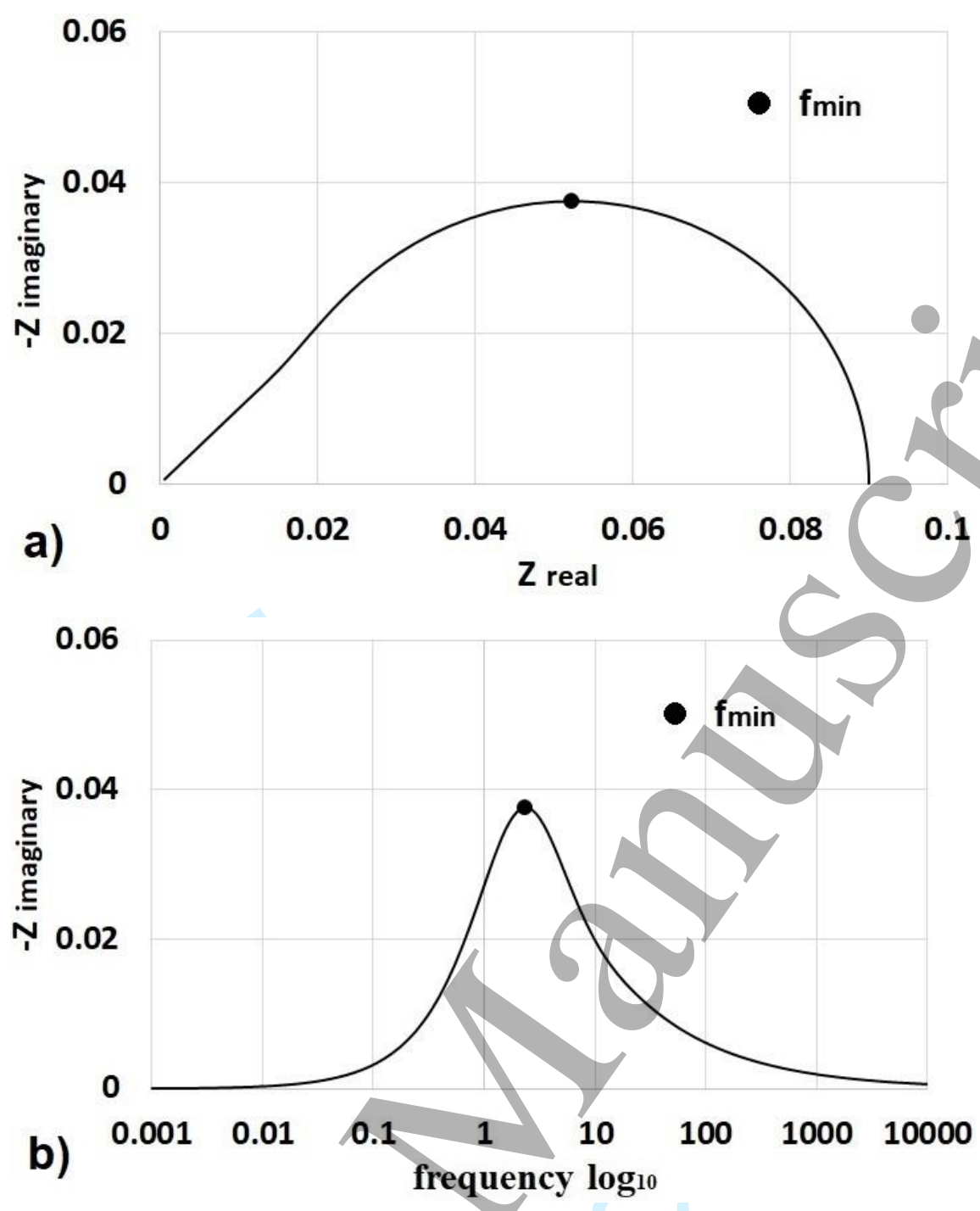

Figure 5. Simulated impedance spectrum for Finite-length Warburg Impedance, a) Impedance response using Eq. 1, b) Imaginary component as a function of frequency calculated through Eq. 16.

\section{Analysis using the Blocked-Diffusion Warburg Impedance}

The mathematical treatment presented in section 3 previously will assist the development of a mathematical expression to calculate the frequency for the transition from diffusion to capacitive responses in the BDW impedance. In a previous study [18], the BDW impedance equation considering frequency dispersion was derived from fundamental diffusion theory and was represented as follows:

$\mathrm{Z}_{\mathrm{BDW}}=\mathrm{R}_{\mathrm{W}} \frac{\operatorname{coth}\left(\mathrm{i} \omega \mathrm{T}_{\mathrm{BDW}}\right)^{0.5 p}}{\left(\mathrm{i} \omega \mathrm{T}_{\mathrm{BDW}}\right)^{0.5 p}}$ 
with

$\mathrm{T}_{\mathrm{BDW}}=\left(\frac{\delta^{2}}{\widehat{\mathrm{D}}}\right)^{\frac{1}{p}}$

where $\mathrm{T}_{\mathrm{BDW}}$ is the power-law time constant distribution from the power law frequency response (frequency dispersion). $\widehat{D}$ is a parameter with units $\mathrm{m}^{2} \mathrm{~s}^{-\mathrm{p}}$ which differs from the conventional diffusion coefficient $D$ with units $\mathrm{m}^{2} \mathrm{~s}^{-1}$ and can be related to anomalous diffusion $[16,25]$. For a given value of $\mathrm{p}$, the characteristic frequency $f_{W}$ can be obtained from the characteristic time constant $\mathrm{T}_{\mathrm{BDW}}$ as such $\mathrm{f}_{\mathrm{W}}=1 /\left(2 \pi \mathrm{T}_{\mathrm{BDW}}\right)$. The characteristic frequency $f_{W}$ is represented in the phase angle of the BDW impedance response as shown in Fig. 2b. $R_{W}$ is similar to Eq. 2. For the specific case that $\mathrm{p}=1$, Eq. 20 represents the conventional BDW impedance [13] and simulates the transition from a $45^{\circ}$ straight-line related to diffusion mechanisms to a vertical line representing a capacitive response as shown in Fig. 2a. Eq. 20 with $\mathrm{p}<1$ (frequency dispersion) simulates a vertical line with slope in the low frequency range as shown in Fig. 2a. Eq. 20 has also been considered to characterize corrosion processes in concentrated solar power (CSP) plants through EIS measurements [26].

Eq. 20 can be expressed as:

$\mathrm{Z}_{\mathrm{BDW}}=\mathrm{R}_{\mathrm{W}} \frac{\operatorname{coth}(\alpha+\mathrm{i} \beta)}{\alpha+\mathrm{i} \beta}$

where $\alpha$ and $\beta$ have been defined in Eqs. 5 and 6 considering $\mathrm{n}=0.5 \mathrm{p}$ and $\mathrm{s}=\omega \mathrm{T}_{\mathrm{BDW}}$

Following the same derivation steps and trigonometric identities expressed in Eqs. 7-12, Eq. 22 can be expressed as:

$\mathrm{Z}_{\mathrm{BDW}}=\frac{\mathrm{R}_{\mathrm{W}}(\alpha-\mathrm{i} \beta)}{\alpha^{2}+\beta^{2}} \frac{\sinh 2 \alpha-\mathrm{i} \sin 2 \beta}{\cosh 2 \alpha-\cos 2 \beta}$

Eq. 23 can be separated into real and imaginary components $\mathrm{Z}_{\mathrm{BDW}}=\mathrm{Z}_{\mathrm{BDW} \_\mathrm{Re}}+\mathrm{i} \mathrm{Z}_{\mathrm{BDW} \_\mathrm{Im}}$ as such: 
$\mathrm{Z}_{\mathrm{BDW}}=\overbrace{\frac{\mathrm{R}_{\mathrm{W}}}{\alpha^{2}+\beta^{2}} \frac{\alpha \sinh 2 \alpha-\beta \sin 2 \beta}{\cosh 2 \alpha-\cos 2 \beta}}^{\mathrm{Z}_{\text {BDW_Re }}}-\mathrm{i} \overbrace{\frac{\mathrm{R}_{\mathrm{W}}}{\alpha^{2}+\beta^{2}} \frac{\alpha \sin 2 \beta+\beta \sinh 2 \alpha}{\cosh 2 \alpha-\cos 2 \beta}}^{\mathrm{Z}_{\text {BDW_Im }}}$

Substituting the parameters $\alpha$ and $\beta$ defined in Eqs. 5 and 6 into the second term on the righthand side of Eq. 24 that represents the imaginary component of the BDW impedance yields:

$\mathrm{Z}_{\mathrm{BDW} \_\mathrm{Im}}=-\frac{\mathrm{R}_{\mathrm{W}}}{\mathrm{s}^{\mathrm{n}}} \frac{\cos (\mathrm{n} \pi / 2) \sin \left\lfloor 2 \mathrm{~s}^{\mathrm{n}} \sin (\mathrm{n} \pi / 2)\right\rfloor+\sin (\mathrm{n} \pi / 2) \sinh \left\lfloor 2 \mathrm{~s}^{\mathrm{n}} \cos (\mathrm{n} \pi / 2)\right\rfloor}{\cosh \left\lfloor 2 \mathrm{~s}^{\mathrm{n}} \cos (\mathrm{n} \pi / 2)\right\rfloor-\cos \left\lfloor 2 \mathrm{~s}^{\mathrm{n}} \sin (\mathrm{n} \pi / 2)\right\rfloor}$

Note that Eq. 25 does not include the imaginary unit $i$, but the numbers generated from Eq. 25 represent the imaginary numbers of the impedance $\mathrm{Z}_{\mathrm{BDW}}$ represented in Eq. 24.

Fig. 6a shows the imaginary components as a function of frequency calculated through Eq. 25. The two responses associated to the diffusion and capacitive processes in the BDW can be observed in Fig. 6a. The slope of the capacitive response depends on the parameter $n$ represented in Eq. 25. The frequency $f_{D-C}$ for the transition from diffusion to capacitive responses can be considered at the interception between the diffusion and capacitive responses as shown in Fig. 6a. The frequency $f_{D-C}$ represented by the imaginary components shown in Fig. 6a can also be represented by the phase-angle of the BDW impedance response as shown in Fig. 6b. A converged solution for $s$ in the function $\mathrm{Z}_{\mathrm{BDW}} \mathrm{Im}(s)$ expressed in Eq. 25 and located at the diffusion-capacitive interception response shown in Fig. 6a is required to be found to estimate the frequency $f D-C$. 
a)

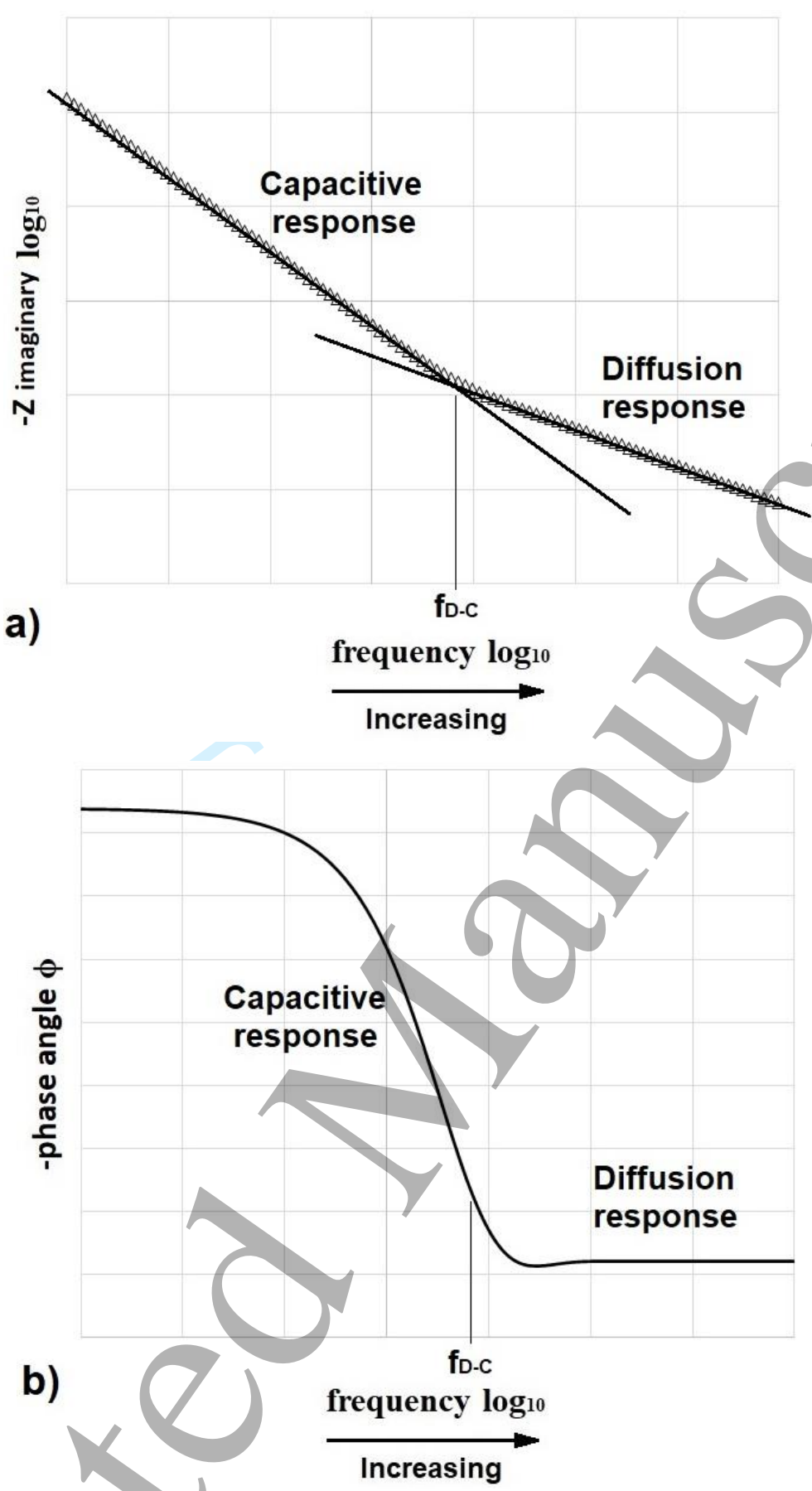

Figure 6. Diffusion and capacitive responses from BDW impedance as a function of frequency, a) Imaginary component, b) phase angle of impedance response

\subsection{Fixed-point method within Newton-Raphson iteration}

The Newton-Raphson iterative method expressed in Eq. 17 was applied to Eq. 25 considering that a converged solution would be achieved at the condition $Z_{B D W_{-} I m}(s)=0$. However, the iteration process converged into an incorrect value for $s$ and it was not related to the diffusioncapacitive interception response shown in Fig. 6a. A modification of the Newton method that 
considers a fixed-point iteration process was used to find a converged solution for $s$ in the diffusion-capacitive interception response shown in Fig. 6a.

During a fixed-point iteration, the sequence $\mathrm{j}=0,1,2, \ldots \mathrm{k}$ in $\mathrm{x}_{\mathrm{j}+1}=\mathrm{g}\left(\mathrm{x}_{\mathrm{j}}\right)$ converges to $r$ because $r$ is a fixed-point of the function $\mathrm{g}(\mathrm{x})$, i.e. $\mathrm{g}(\mathrm{r})=\mathrm{r}$.

Newton's method expressed in Eq. 17 is a form of Fixed-point iteration:

$g(x)=x-\frac{F(x)}{F^{\prime}(x)}$

where $g(r)=r$ because $F(r)=0$

The derivative of Eq. 26 can be expressed as:

$\mathrm{g}^{\prime}(\mathrm{x})=\frac{\mathrm{F}(\mathrm{x}) \mathrm{F}^{\prime \prime}(\mathrm{x})}{\left[\mathrm{F}^{\prime}(\mathrm{x})\right]^{2}}$

The iterative process of Eq. 27 can be expressed as:

$\mathrm{x}_{\mathrm{j}+1}=\frac{\mathrm{F}\left(\mathrm{x}_{\mathrm{j}}\right) \mathrm{F}^{\prime \prime}\left(\mathrm{x}_{\mathrm{j}}\right)}{\left[\mathrm{F}^{\prime}\left(\mathrm{x}_{\mathrm{j}}\right)\right]^{2}}$

The use of the Newton method with the fixed-point iteration expressed in Eq. 28 can be applied to the function expressed in Eq. 25 to obtain a converged solution for $s$ at the diffusioncapacitive interception response shown in Fig. 6a, as such:

$\mathrm{S}_{\mathrm{j}+1}=\left[\frac{\mathrm{z}_{\mathrm{BDW} \_\mathrm{Im}}\left(\mathrm{s}_{\mathrm{j}}\right) \mathrm{Z}_{\mathrm{BDW} \_ \text {Im }}{ }^{\prime \prime}\left(\mathrm{s}_{\mathrm{j}}\right)}{\left(\mathrm{z}_{\mathrm{BDW} \_I \mathrm{Im}^{\prime}}{ }^{\prime}\left(\mathrm{s}_{\mathrm{j}}\right)\right)^{2}}\right]^{1 / p}$

where $\mathrm{j}=0,1,2, \ldots \mathrm{k}$ is the iterative sequence for a converged solution $s_{k}$, the exponent $1 / p$ calculates a converged solution of $s_{k}$ for any given value of $p$ (change in slope of capacitive response shown in Fig. 6a). Noting that the same exponent 1/p has been defined in Eq. 21 related to the power-law time constant $\mathrm{T}_{\mathrm{BDW}}$ in BDW with frequency dispersion. The exponent 
$1 / \mathrm{p}$ in the time constant of the BDW has been associated to anomalous diffusion $[16,25]$, $\mathrm{Z}_{\mathrm{BDW} \_\mathrm{Im}}{ }^{\prime}$ is the first derivative and $\mathrm{Z}_{\mathrm{BDW} \_\mathrm{Im}}{ }^{\prime \prime}$ is the second derivative of Eq. 25 with respect to the variable $s$.

The iteration process expressed in Eq. 29 can be written in a script in Matlab® software. As previously mentioned in section 3.1, Matlab® software allows symbolic programming and computing and allows to obtain the derivative of symbolic functions using defined syntaxes. The number of iterations will depend on the proximity of the initial guest $s_{0}$ to the converged solution $s_{k}$. The converged solution for $s_{k}$ calculated through Eq. 29 only changes with the parameter $p$ regardless the value of $R_{W}$ and $\mathrm{T}_{\mathrm{BDW}}$ in Eq. 25 as the converged solution for the variable $s$ corresponds to the nondimensional expression $\mathrm{s}=\omega \mathrm{T}_{\mathrm{BDW}}$. Any value of $R_{W}$ is required during the iteration process, but the value of $R_{W}$ only increases in magnitude the impedance response shown in Fig. 6a and does not change the slope of the diffusion and capacitive responses shown in Fig. 6a. Values for $p=1$ (no frequency dispersion) and $p=$ 0.78 (frequency dispersion) considering that $\mathrm{n}=0.5 \mathrm{p}$ have been considered for the estimation of the converged solution $s_{k}$ through the iteration process from Eq. 29. Table II shows the iteration results for $\mathrm{p}=1$ and using the iteration calculation process expressed in Eq. 29. Table III shows the iteration result for $\mathrm{p}=0.78$. The iteration results expressed in Table II and III and using Eq. 29 were calculated using a script written in Matlab® software as shown in Appendix A2. 


\begin{tabular}{|c|c|c|c|}
\hline $\mathrm{j}$ & $\mathrm{s}_{\mathrm{j}}$ & $\mathrm{s}_{\mathrm{j}+1}$ & error \% \\
\hline 0 & 1 & 3.09133 & 34.623 \\
\hline 2 & 3.94928 & 4.35848 & 7.825 \\
\hline 4 & 4.55406 & 4.64666 & 1.731 \\
\hline 9 & 4.72459 & 4.72667 & 0.038 \\
\hline 14 & 4.72841 & $\mathbf{4 . 7 2 8 4 6}$ & $8.542 \mathrm{e}-04$ \\
\hline
\end{tabular}

Table III. Iteration process for $\mathrm{p}=0.78$.

Fig. 7 shows different converged values for $s_{k}$ considering different values of $p$ during the iteration process in Eq. 29 and using the Matlab script shown in Appendix A2. The resulting values of $s_{k}$ increase with decreasing $p<1$. The converged solution expressed in Table II for $\mathrm{p}=1$ resulted in $\mathrm{s}_{\mathrm{k}}=3.8875$ and for $\mathrm{p}=0.78$ resulted in $\mathrm{s}_{\mathrm{k}}=4.7285$ as expressed in Table III. These results lie in the curve shown in Fig. 7.

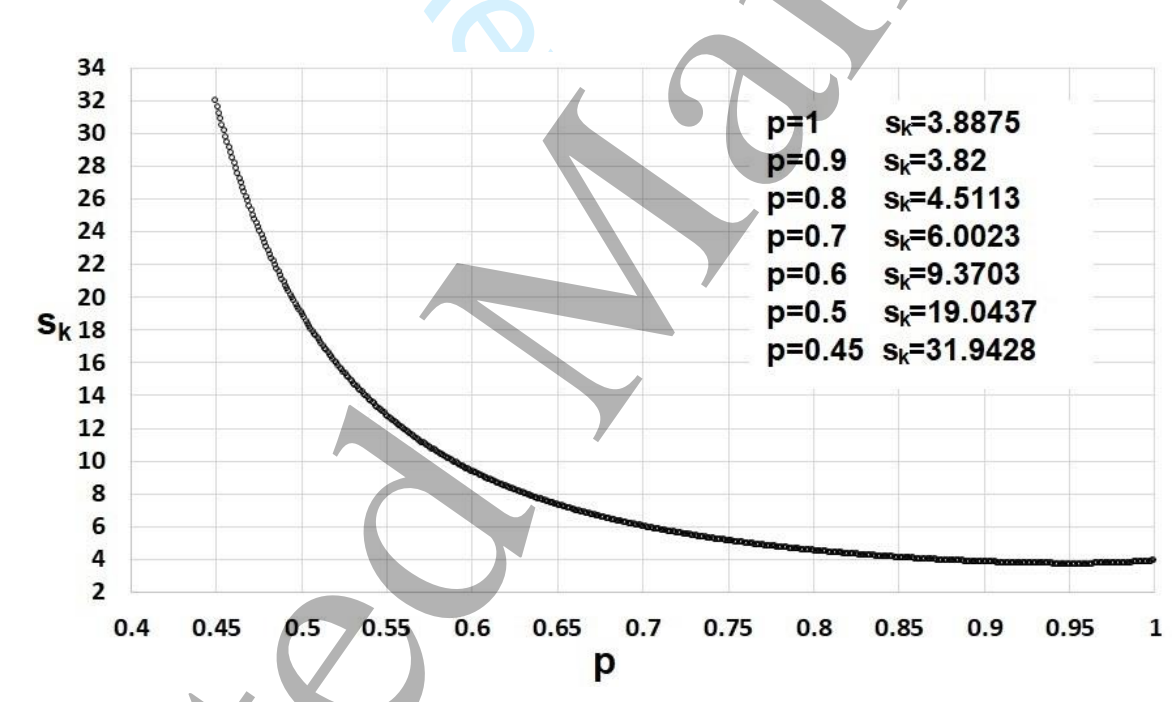

Figure 7. Converged values for $\mathrm{s}_{\mathrm{k}}$ using Eq. 29

\subsection{Frequency and time constant for diffusion-capacitive transition response}

Considering that $\mathrm{s}=\omega \mathrm{T}_{\mathrm{BDW}}$, it is possible to define a mathematical expression for the converged solution $s_{k}$ (Fig. 7) and the frequency $f_{D-C}$ for transition from diffusion to capacitive responses in the BDW impedance as such:

$$
f_{D-C}=\frac{s_{k}}{2 \pi T_{B D W}}
$$


The time constant $\mathrm{T}_{\mathrm{BDW}}$ represented in Eq. 21 represents the time constant when $60 \%$ of charge carriers have been accumulated in the blocked-interface. This was discussed and graphically represented in terms of characteristic frequency $f_{W}=1 /\left(2 \pi T_{B D W}\right)$ and the phase angle of the BDW impedance as shown Figs. $2 \mathrm{~b}$ and 3. From Eq. 30, it is possible to define a relation between the characteristic time constant $\mathrm{T}_{\mathrm{BDW}}$ and the time constant $T_{D-C}$ for the diffusioncapacitive transition response in the BDW as such:

$\mathrm{T}_{\mathrm{D}-\mathrm{C}}=\frac{\mathrm{T}_{\mathrm{BDW}}}{\mathrm{s}_{\mathrm{k}}}$

Eq. 31 can be redefined from Eq. 21 as:

$\mathrm{T}_{\mathrm{BDW}}=\mathrm{s}_{\mathrm{k}}\left(\frac{\delta_{s}{ }^{2}}{\widehat{\mathrm{D}}_{s}}\right)^{\frac{1}{p}}$

where $\widehat{D}_{s}$ is a parameter with units $m^{2} s^{-p}$ which differs from the conventional diffusion coefficient $D_{S}$ with units $m^{2} s^{-1}$ and is related to anomalous diffusion [16]. $\widehat{D}_{s}$ will be coined as "diffusion constant" for simplicity. $\widehat{D}_{S}$ is the diffusion constant related to the frequency $f_{D-C}$ and time constant $\mathrm{T}_{\mathrm{D}-\mathrm{C}}$ during the diffusion-capacitive transition response. Peng et al. [22] related the ionic chemical diffusion coefficient of lead halide perovskite materials of solar cells with the frequency during the diffusion-capacitive transition response in the BDW response. $\widehat{D}_{S}$ is higher than the diffusion constant $\widehat{D}$ of charge carriers in the blocked-interface represented in the characteristic time constant $\mathrm{T}_{\mathrm{BDW}}$ in BDW impedance (Eq. 20). Hence $\mathrm{T}_{\mathrm{BDW}}>\mathrm{T}_{\mathrm{D}-\mathrm{C}}$ or $f_{W}<\mathrm{f}_{\mathrm{D}-\mathrm{C}}$ as shown in Figs. $2 \mathrm{~b}$ and 3. A higher value of $\widehat{D}_{s}$ compared to $\widehat{D}$ could be related to the fact that a higher concentration of solid-particles is distributed across the electrode compared to the concentration of solid-particles interacting within the blocked-interface. The solid-phase electroactive material and the blocked-interface (current collector) in the electrode have been depicted in Fig. 1. $\delta_{s}$ can be related to the actual geometry of the particle which is less than the total diffusion distance $\delta$ of ions moving from the solid-phase particles to the blocked-interface (e.g. current collector) in the electrode as shown in Fig. 1. 


\subsubsection{Estimation of $f_{D-C}$ in the BDW impedance}

For the specific case when $\mathrm{p}=1$, Eq. 30 calculates the frequency $f_{D-C}$ for the transition from a $45^{\circ}$ straight-line (diffusion) to a vertical line (capacitive) in the Warburg impedance response, as shown in Fig. 2a. Under this condition, Eq. 30 can be expressed as:

$\mathrm{f}_{\mathrm{D}-\mathrm{C}_{\mathrm{p}=1}}=\frac{3.8875}{2 \pi \mathrm{T}_{\mathrm{BDW}}}$

where the pre-factor 3.8875 represents the converged solution of $s_{k}$ in the iterative process of Eq. 29 and expressed in Table II and shown in Fig. 7. Eq. 33 implies that the frequency $f_{D-C}$ during the transition from diffusion to capacitive response is a factor 3.8875 higher than the characteristic frequency related to the characteristic time constant $\mathrm{f}_{\mathrm{W}}=1 /\left(2 \pi \mathrm{T}_{\mathrm{BDW}}\right)$ represented in the BDW impedance, Eq. 20. The frequencies $f_{D-C}$ and $f_{W}$ have been depicted in the Nyquist and Bode plots shown in Fig. 2. ZView software (Scribner Associates, Inc.) considers a pre-factor of 2.53 (similar to Eq. 19 for FLW impedance) instead of the pre-factor 3.8875 in Eq. 33 for calculation of $f_{D-C}$ in the BDW. But no analysis or justification is described in the documentation of the ZView software for using such a pre-factor of 2.53 in the BDW impedance. In addition, when $\mathrm{p}=1$ in the BDW impedance, the time constant $T_{D-C}$ expressed in Eq. 31 is a factor 3.8875 smaller than the characteristic time constant $\mathrm{T}_{\mathrm{BDW}}$ considered in the BDW.

When $\mathrm{p}=0.78$ in the BDW, the frequency $f_{D-C}$, calculated through Eq. 30 and with the converged solution from Table III and shown in Fig. 7, is a factor 4.7285 bigger than the characteristic frequency $f w$.

Fig. 8a shows the impedance response of the BDW calculated with Eq. 20 and the parameters $\mathrm{R}_{\mathrm{W}}=0.4, \mathrm{~T}_{\mathrm{BDW}}=0.1 \mathrm{p}=1$ and $\mathrm{p}=0.78$ and a range of frequencies $\mathrm{f}=10 \mathrm{kHz}-$ $0.001 \mathrm{~Hz}$. Fig. $8 \mathrm{~b}$ shows the angle phase of the BDW impedance response from Fig. 8a. The frequency $f_{D-C}$ during the diffusion-capacitive transition response calculated through Eq. 30 and shown in Fig. $8 \mathrm{~b}$ for $p=1$ resulted in $6.187 \mathrm{~Hz}$ and for $p=0.78$ resulted in $7.526 \mathrm{~Hz}$. 

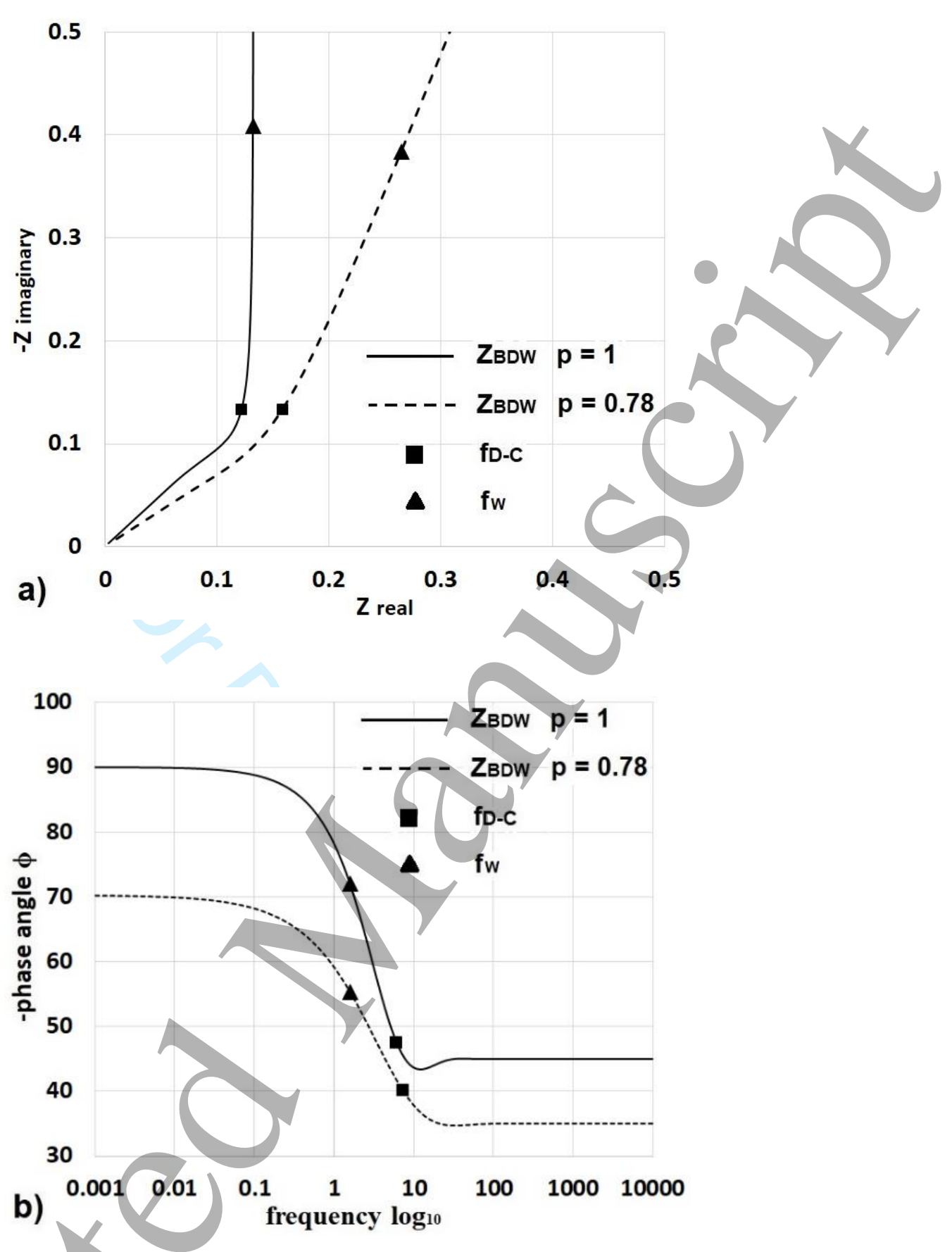

Figure 8. Simulated impedance spectrum for Blocked-diffusion Warburg Impedance with $\mathrm{R}_{\mathrm{W}}=0.4, \mathrm{~T}_{\mathrm{BDW}}=0.1 \mathrm{p}=1$ and $\mathrm{p}=0.78$; the frequency $f_{D-C}$ calculated with Eq. 30 for $\mathrm{p}=1$ is $6.187 \mathrm{~Hz}$ and for $\mathrm{p}=0.78$ is $7.526 \mathrm{~Hz}$, a) Impedance response calculated from Eq. 20, b) phase angle of impedance response

\subsubsection{Estimation of $f_{D-C}$ in the BDW impedance response of a Li-ion battery}

In a previous study [32] an EEC comprising a BDW was fitted to EIS measurements carried out in a Li-ion battery as shown in Fig. 9. The contribution of the BDW on the overall EIS 
response was predicted at frequencies from $0.01 \mathrm{~Hz}$ to $0.002 \mathrm{~Hz}$ as shown in Fig. 9. The BDW was related to the impedance diffusion response of Lithium ions $\mathrm{Li}^{+}$on the solid-phase of the electroactive material in the electrode. The impedance response from $1 \mathrm{~Hz}$ to $0.01 \mathrm{~Hz}$ was related to the impedance diffusion response of $\mathrm{Li}^{+}$on the solution-phase of the porous electrode and was predicted through a FLW. More details about the EIS response of the Li-ion battery and the representation of the electrochemical mechanisms at different frequencies in the impedance spectrum can be found in the previous study [32]. Fig. 9 demonstrates that it is not possible to estimate through a graphical interpretation of the impedance spectrum the frequency $f_{D-C}$ during the transition from diffusion response to capacitive response of the BDW. The BDW response at frequencies from $0.01 \mathrm{~Hz}$ to $0.002 \mathrm{~Hz}$ is overlapped with other mechanisms represented in the range of $1 \mathrm{~Hz}$ to $0.01 \mathrm{~Hz}$ in the impedance spectrum.

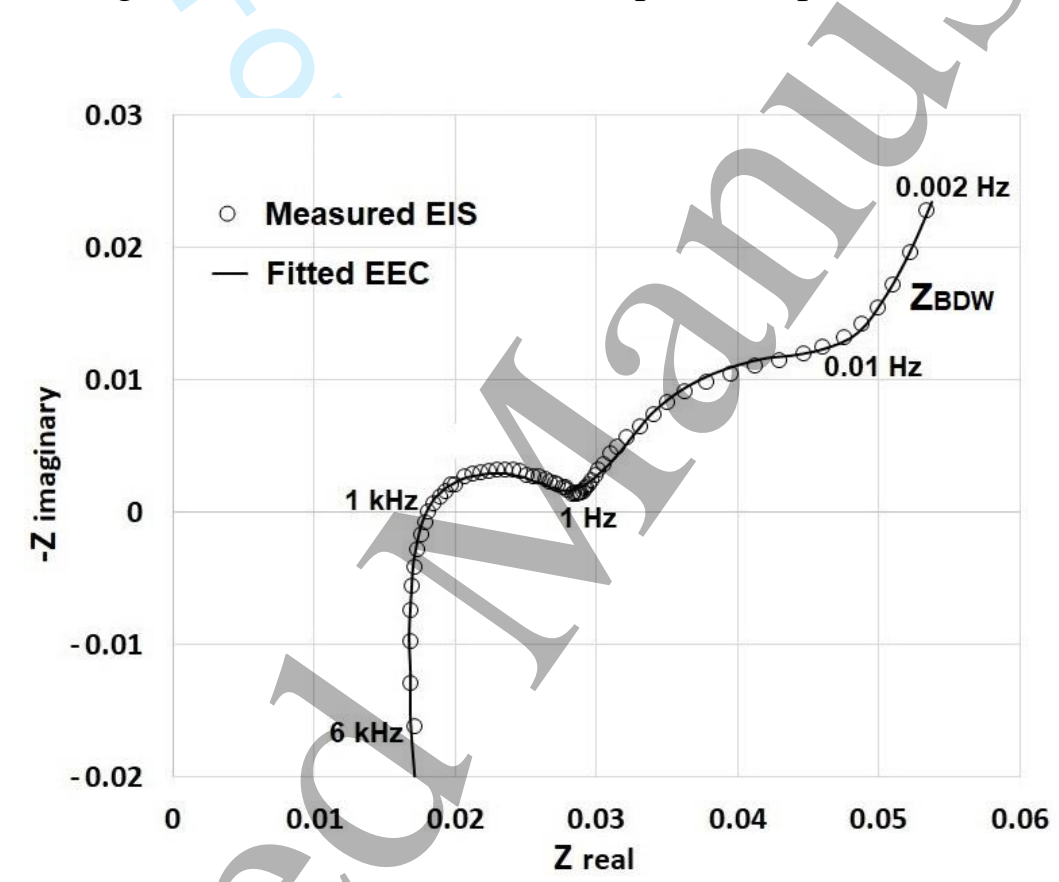

Figure 9. EIS measurements in a Li-ion battery fitted with an EEC comprised of BDW [32].

The values for $\mathrm{R}_{\mathrm{W}}=0.001, \mathrm{~T}_{\mathrm{BDW}}=1.4, \mathrm{p}=0.78$ representing the $\mathrm{BDW}$ in the EEC from $\mathrm{a}$ previous study [32] have been considered for calculation of the frequency $f_{D-C}$ in the diffusioncapacitive transition response. Fig. 10a shows the impedance spectrum of the BDW calculated with Eq. 20 and the parameters $\mathrm{R}_{\mathrm{W}}=0.001, \mathrm{~T}_{\mathrm{BDW}}=1.4 \mathrm{p}=0.78$ estimated in a previous study [32] and with a range of frequencies $\mathrm{f}=10 \mathrm{kHz}-0.001 \mathrm{~Hz}$. Fig. 10b shows the angle phase of the BDW impedance response from Fig. 10a. The frequency $f_{D-C}$ during the transition from the diffusion to the capacitive response calculated through Eq. 30 and the iterative process from Eq, 29 and shown in Fig $10 \mathrm{~b}$ resulted in $0.537 \mathrm{~Hz}$. The frequency $f_{D-C}$ and time constant 
$T_{D-C}$ are related to the diffusion of $\mathrm{Li}^{+}$on the solid-phase in the electroactive material of the Li-ion battery. It is possible to estimate the diffusion-length of solid-particles $\delta_{s}$ from the time constant $T_{D-C}$ by considering diffusion coefficients in solid-particles reported in other studies. Gruet et al. [33] developed an impedance model for porous electrodes in Li-ion batteries and considered diffusion coefficients reported in the study of Smith et al. [34]. The diffusion coefficients were estimated using a higher order CFD model [35].

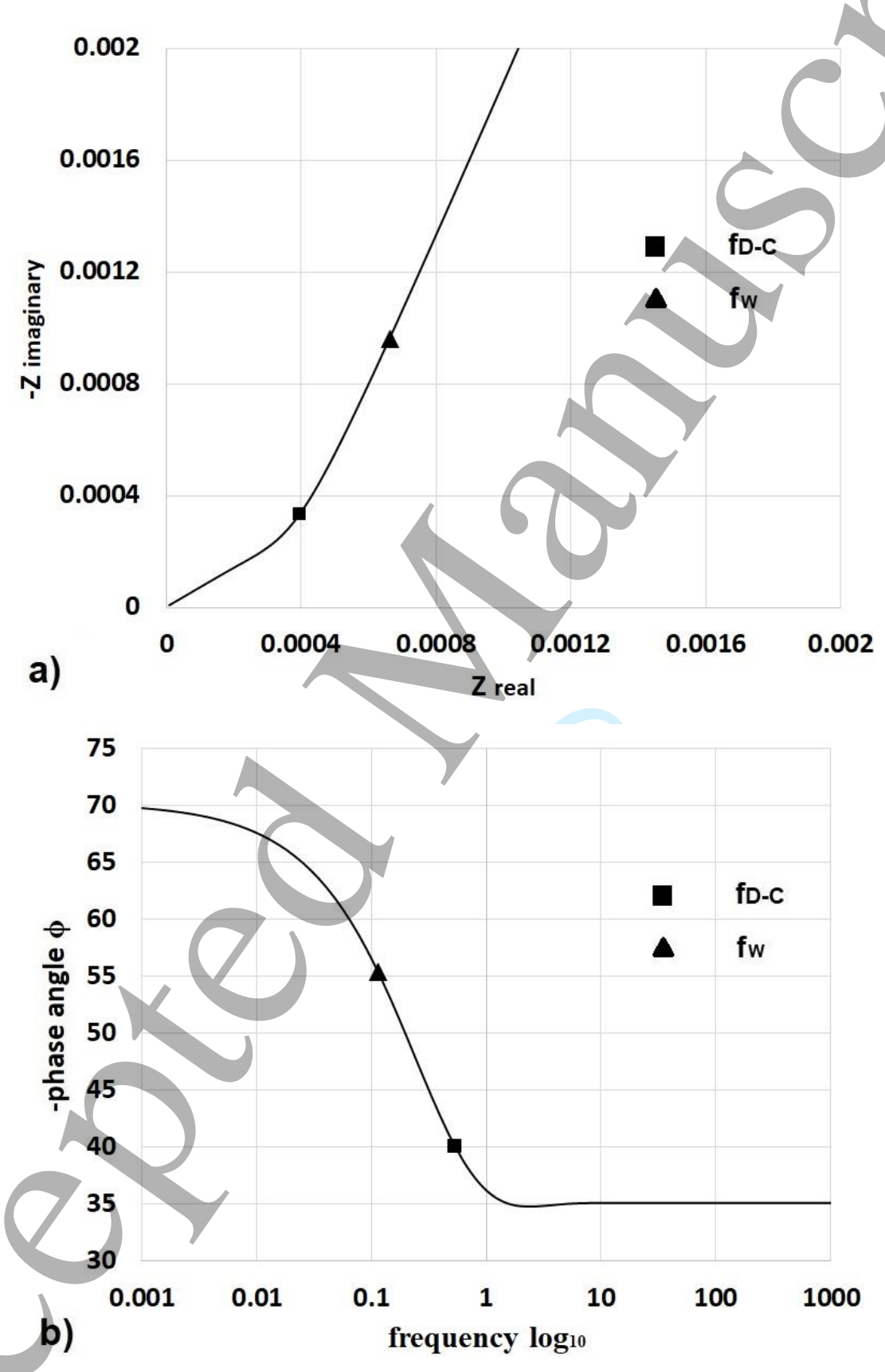

Figure 10. Simulated impedance spectrum for Blocked-diffusion Warburg Impedance with $\mathrm{R}_{\mathrm{W}}=0.001, \mathrm{~T}_{\mathrm{BDW}}=1.4 \mathrm{p}=0.78$ estimated from a previous study [32] in EIS measurements 
in a Li-ion battery. Frequency $f_{D-C}$ calculated with Eq. 30 resulted in a frequency of $0.537 \mathrm{~Hz}$, a) Impedance response calculated with Eq. 20, b) phase angle of impedance response.

\section{Estimation of $f_{D-C}$ in the EIS response of a NiMH battery at different SoC}

In a previous study [18], an EEC comprised of a BDW was fitted to EIS measurements carried out in a NiMH battery at different SoC. The EIS measurements were carried out in a 9.6V rechargeable NiMH AA 2500mAh, battery pack. The NiMH battery was initially fully charged and then it was discharged at a constant current of $3.3 \mathrm{~A}$ until a voltage of 9.18 Volts was measured. The load was disconnected and the battery pack was left at open circuit (equilibrium condition) for one hour. Thereafter, EIS measurements were carried out at open circuit voltage $(\mathrm{OCV})$ under a potentiostatic configuration and with an a.c. perturbation of $10 \mathrm{mV}$ to ensure steady-state conditions during the impedance measurements and at frequencies from $20 \mathrm{kHz}$ to $0.005 \mathrm{~Hz}$. The same procedure was carried out for EIS measurements at OCV of 8.4, and 8 Volts. Further information about the EIS measurements carried out in the NiMH battery can be found in the authors' previous study [36]. An EEC comprised of a BDW considering frequency dispersion was fitted to the EIS-NiMH battery measurements at OCV values of 9.18, 8.4, and 8V. Fig. 11 shows a comparison between the EIS-NiMH measurements and the impedance response predicted by the EEC with BDW. The semicircle at high frequencies is attributed to the rate of charge transfer during the redox reaction and the straight-line with slope at low frequency was attributed to nonhomogeneous accumulation of charge (capacitive response) in the blocked-interface (current collector) of the electrode. The impedance response (straightline) predicted by the BDW at low frequencies was overlapped with the impedance response (semicircle) at high frequencies predicted by a Randles circuit. Therefore, it is not possible to accurately estimate through a graphical interpretation on the Nyquist plot the frequency $f_{D-C}$ during the transition from diffusion to capacitive response in the BDW with decreasing SoC in the NiMH battery. 


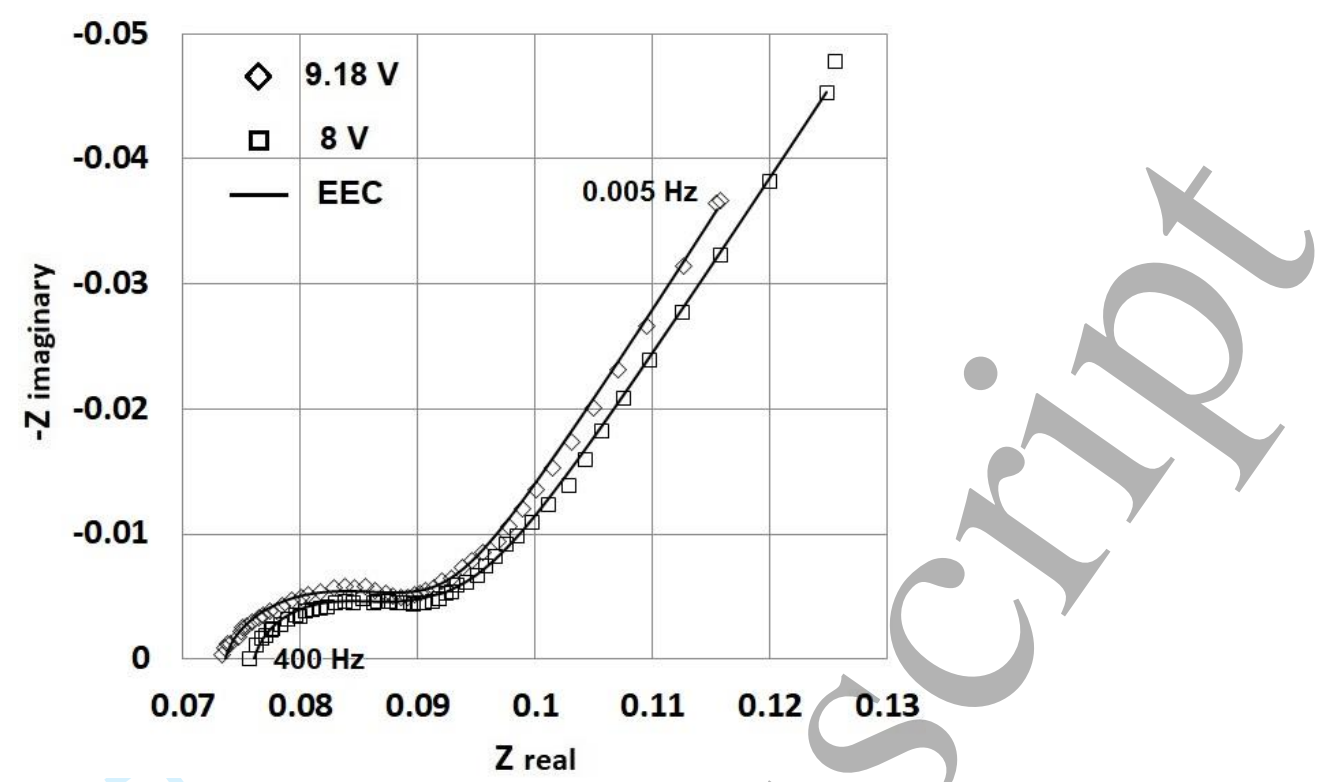

Figure 11. EIS measurements in a NiMH battery fitted with an EEC comprised of a BDW [18].

In this study, the reported values of $R_{W}, \mathrm{~T}_{\mathrm{BDW}}, p$ from the BDW extracted from the EIS response of the NiMH battery are considered to simulate the BDW impedance at different $\mathrm{OCV}$ values. Table IV shows the parameters of the BDW at different OCV. In addition, Table IV shows the frequency $f_{D-C}$ and time costant $T_{D-C}$ for diffusion-capacitive transition response calculated through Eqs. 30, 31 and the iterative process from Eq. 29. The resulting values of the parameter $s_{k}$ at different values of $p$ lie on the curve shown in Fig. 7.

\begin{tabular}{|c|c|c|c|c|c|c|}
\hline $\mathrm{OCV}$ & $R_{W}, \Omega$ & $\mathrm{T}_{\mathrm{BDW}}, \mathrm{s}$ & $\mathrm{p}$ & $f_{D-C}, \mathrm{~Hz}$ & $T_{D-C}, \mathrm{~s}$ & $s_{k}$ \\
\hline 9.18 & 0.0191 & 8.409 & 0.62 & 0.1596 & 0.9974 & 8.4307 \\
\hline 8.4 & 0.0211 & 9.446 & 0.606 & 0.1528 & 1.0416 & 9.0687 \\
\hline 8 & 0.0271 & 10.06 & 0.612 & 0.139 & 1.1452 & 8.7845 \\
\hline
\end{tabular}

Table IV. Parameters of BDW at different open-circuit voltage reported in a previous study [18] and calculated with Eqs. 29, 30,31.

Fig. 12 shows the simulated BDW impedance response predicted by Eq. 20 at frequencies from $10 \mathrm{kHz}$ to $0.001 \mathrm{~Hz}$ and with the parameters $R_{W}, \mathrm{~T}_{\mathrm{BDW}}, p$ shown in Table IV at different OCV. The real component $Z_{\text {real }}$ of the BDW impedance response is increased with decreasing OCV as shown in Fig. 12a. The magnitude of the straight-line at low frequencies increases with decreasing OCV as shown in Fig. 11. The frequency $f_{D-C}$ for diffusion-capacitive transition 
response in the BDW impedance decreases with decreasing OCV as shown in Table IV and shown in the phase angle of the Bode plot from Fig. 12b.

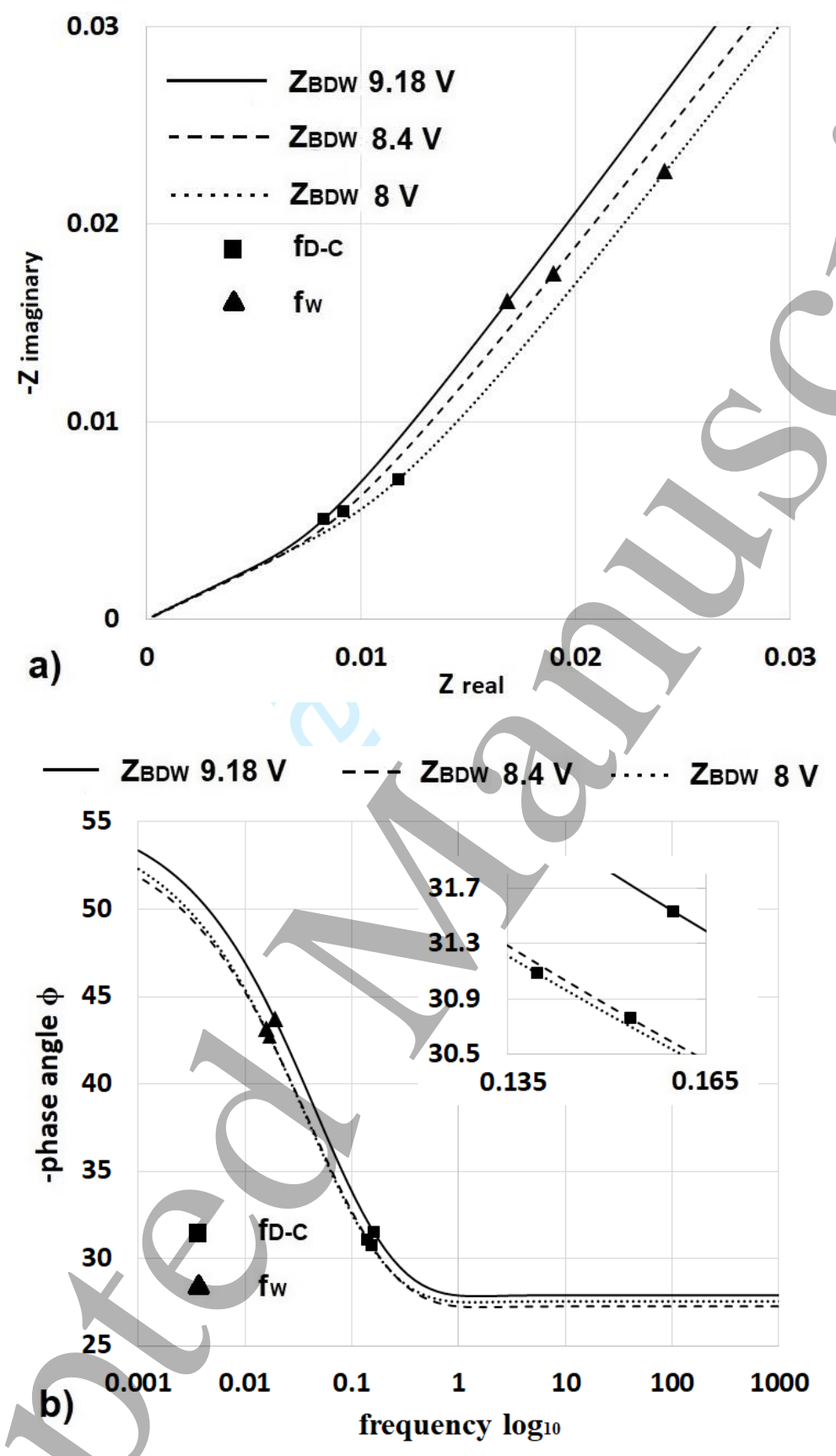

Figure 12. Simulated impedance spectrum for BDW with parameters estimated from a previous study [18] in EIS measurements in a NiMH battery. Frequency $f_{D-C}$ calculated with Eq. 30, a) Impedance response calculated with Eq. 20, b) phase angle of impedance response.

The magnitude of the diffusion impedance response shown in Fig. 12 is directly proportional to the diffusion resistance $R_{W}$. The time constant $T_{D-C}$ for diffusion-capacitive transition 
response is smaller than the characteristic time constant $\mathrm{T}_{\mathrm{BDW}}$ represented in the BDW equation (Eq. 20) as shown in Table IV. The time constants $\mathrm{T}_{\mathrm{BDW}}$ and $T_{D-C}$ increased with decreasing OCV as shown in Table IV. The diffusion time constant $T_{D-C}$ is directly proportional to the diffusion length $\delta_{s}$ and inversely proportional to the diffusion constant $\widehat{D}_{s}$. The solid-phase electroactive material is consumed during the discharge of the battery [37], therefore the diffusion length $\delta_{s}$ in the solid-phase electroactive material of the electrode decreases with decreasing SoC in the batteries. The diffusion constant $\widehat{D}_{S}$ has a bigger effect on the BDW impedance [38] and is reduced with decreasing OCV. Hence an increase in time constant $T_{D-C}$ with decreasing OCV is observed in Table IV. The hydrogen diffusion coefficient in NiMH electrodes decrease with decreasing the battery SoC [39]. The diffusion constant $\widehat{D}_{S}$ is dependent on diffusion length $\delta_{s}$ as a reduction in diffusion length $\delta_{s}$ (solid-phase electroactive material) yields a reduction of ion concentration.

The time constant $T_{D-C}$ increased by $4.2 \%$ when discharging the NiMH battery from 9.18 to $8.8 \mathrm{~V} . T_{D-C}$ increased by $9 \%$ when discharging the NiMH battery from $8.8 \mathrm{~V}$ to $8 \mathrm{~V}$. A lower diffusion constant $\widehat{D}_{s}$ yields a higher increase in $T_{D-C}$. The BDW impedance responses shown in Fig. 12 were extracted from the EIS measurements shown in Fig. 11 and carried out in a NiMH battery pack of 8 cells with a nominal voltage of 9.6V [18,36]. A lower diffusion constant $\widehat{D}_{S}$ and a higher $T_{D-C}$ in EIS measurements of the NiMH battery pack can be related to the fact that EIS measurements at $8 \mathrm{~V} \mathrm{OCV}$ were carried out close to the nonlinear discharge response (knee of discharge) of the NiMH battery. The knee of discharge feature during the battery discharge response is commonly apparent at voltages lower than 1V/cell [40]. This study can assist other theoretical studies for the interpretation of impedance diffusion of electroactive materials at low ion concentration in which transport of charge carriers from different diffusion interphases in the electrode may play an important role $[15,41]$.

\section{Discussion}

The analysis of this study has been focused on the BDW equation that corresponds to the diffusion impedance response of an electrode with planar particles. BDW impedances considering diffusion of ions in the electrodes with different solid-particle geometry e.g. spherical, cylindrical have been considered for the analysis of EIS measurements in Li-ion batteries $[42,43]$. This modelling framework could assist the estimation of the frequency and time constant for the diffusion-capacitive transition response in EIS measurements carried out 
in electrodes with different solid-particle geometry. The methodology developed in this study and presented in Section 4 can be applied to the different BDW impedance equations considering different solid-particle geometry in the electrode as the diffusion-capacitive transition response at low frequencies is represented in the impedance response of battery electrodes with different solid-particle geometry [15]. A recent study reported by Li et al. [44] demonstrated that voltage oscillations during the galvanostatic discharge/charge in Li-ion batteries can be attributed to the multi-particle phase-separation reaction. The voltage oscillation period could be a function of particle size distribution in the electrode. It is possible to extend this EIS study by comparing the diffusion time constant of charge-carriers in the solid-phase of electrodes with different particle geometry. This information can be relevant to assess the dynamic response of a battery management system during the charge/discharge of batteries with different solid-particle electrode structure.

The characteristic frequency $f w$ on the phase-angle of the BDW impedance shown in Fig. 3 indicates that a total of $60 \%$ of the charge carriers has been accumulated before reaching a full accumulation (90 degree in phase angle) of charge in the blocked-interface of the electrode. The characteristic frequency $f_{W}$ and frequency $f_{D-C}$ for diffusion-capacitive transition response in the BDW impedance response will change at different battery SoC. A variation of the phase angle related to the characteristic frequency $f_{W}$ and represented in the BDW impedance response with decreasing SoC in a battery is expected as well. This has been demonstrated in Section 5 of the manuscript/when analysing the BDW impedance response from EIS measurements in a NiMH battery at different SoC. A change in the diffusion time constant which is related to the characteristic frequency of the diffusion impedance at low frequencies has been reported in EIS measurements of Li-ion batteries at different SoC [43]. The diffusion constant $\widehat{D}$ represented in the characteristic time constant $\mathrm{T}_{\mathrm{BDW}}$ of the BDW (Eq. 20) is related to the concentration of charge carriers $(60 \%)$ approaching a full accumulation of charge $(100 \%)$ in the blocked-interface (e.g. current collector) in the electrode. When the parameter $p$ related to frequency dispersion in the BDW was reduced $p<1$, a vertical line with slope at low frequencies was predicted, as shown in Fig. 2a. Song et al. [15] simulated the effect of particle size and different geometry distribution of solid particles on EIS of Li-ion batteries. The slope of the vertical line at low frequencies attributed to the blocked-interface of the BDW decreased with heterogenous particle size distribution. The relation between the parameter $s_{k}$ predicted from the Newton-Raphson iteration (Eq. 29) combined with the estimation of diffusion 
constant $\widehat{D}_{s}[20,22,29,45]$ in Eq. 32 can provide an estimation of the actual diffusion length $\delta_{s}$ (geometry) in the solid-particles of the electrode. Bandara et al. [46] estimated the diffusion coefficient in polymer electrolytes of Li-ion batteries using EIS measurements and a mathematical function for dielectric analysis in electrolytes. Meyers et al. [47] demonstrated that if the diffusion coefficient is increased in the diffusion impedance of a porous electrode composed of spherical intercalation particles, the impedance response approaches the limit of purely capacitive behaviour (straight-line at low frequencies). The diffusion constant $\widehat{D}_{s}$ represented in Eq. 32 is related to the concentration of charge carriers in the solid-phase electroactive material of the electrode. An increase in the diffusion constant $\widehat{D}_{S}$ yields a decrease in the time constant $T_{D-C}$ and an increase in the frequency $f_{D-C}$ for the transition from diffusion to capacitive response in the BDW impedance response. The diffusion constant is also inversely proportional to the diffusion resistance $R_{W}$. In addition, an increase in the diffusion constant $\widehat{D}_{S}$ will reduce the diffusion response of the BDW at high frequencies and the BDW impedance response approaches the limit of purely capacitive behaviour (straightline at low frequencies) as reported by Meyers et al. [47]. The diffusion constant $\widehat{D}_{s}$ will change for different geometry of the solid particles (planar, cylindrical, spherical) and for heterogenous geometry distribution of solid particles in the electrode. A heterogenous solid particle distribution could not only yield frequency dispersion in EIS measurements $p<1$, but also could yield a reduction in the plateau voltage which is related to a decrease the miscibility gap (metastable uniform compositions) between the two-phases in the particles [48].

It has been reported changes in the impedance response of batteries occur at different SoC and $\mathrm{SoH}[49,50,51]$. It has also been reported that a change in the frequency represented in the Nyquist plot and associated to the intersection between a semicircle at high frequencies and the BDW response at low frequencies occur in the impedance response of a Li-ion battery at different Li concentrations [15]. Jiang et al. [52] reported a nonlinear increase in ohmic and charge transfer resistances estimated from EIS measurements in Li-ion batteries with different ageing states. Martínez et al. [43] studied the electrochemical mechanisms from metal-hydride electrodes used in NiMH batteries through EIS measurements and using an impedance model representing a porous electrode. The results demonstrated a variation of the hydrogen diffusion time constant estimated from the EIS measurements of the electrodes at different SoC, the hydrogen diffusion time constant is related to the characteristic frequency of the hydrogen diffusion impedance. Capron et al. [53] estimated the solid-state diffusion coefficient in Nickel 
Manganese Cobalt oxide (NMC) electrodes using EIS measurements. The solid-state diffusion coefficient estimated from the SDW impedance decreased during long-term cycling of the cell. Based on the studies reported in the literature, there is a correlation in the electrochemical and diffusion mechanisms in batteries at different $\mathrm{SoC}$ and $\mathrm{SoH}$, but some physical parameters are more affected than others, e.g. a higher increase in charge transfer resistance than ohmic resistance estimated from EIS measurements in Li-ion batteries with different ageing states has been reported [52]. Further EIS analysis is still required to be carried out to assess the change in diffusion mechanisms (e.g. diffusion coefficient of charge carriers in electrodes with different solid-particle geometry) estimated through BDW impedances and during the battery lifetime. This could assist the development of algorithms in an energy management system to minimise losses during the dynamic performance of a battery management system [49].

\section{Conclusions}

The frequency $f_{D-C}$ and time constant $T_{D-C}$ during the transition from diffusion response to capacitive response in the BDW impedance has been calculated through the Newton-Raphson method. The characteristic time constant $\mathrm{T}_{\mathrm{BDW}}$ represented in the BDW impedance equation is related to the rate of accumulation of charge carriers in the blocked-interface of the electrode and differs from the time constant $T_{D-C}$ during the diffusion-capacitive transition response in the BDW. The BDW impedance response with frequency dispersion $(p<1$ in the BDW impedance equation) can be related to a heterogenous solid particle distribution in the electroactive material of the electrode. A change in the time constant $T_{D-C}$ and frequency $f_{D-C}$ for the diffusion-capacitive transition response in the BDW can be attributed to a change in the diffusion constant $\widehat{D}_{S}$ of charge carriers in the solid-phase of particles in the electrode. Estimation of frequency $f_{D-C}$ and time constant $T_{D-C}$ from EIS measurements in NiMH and Li-ion batteries has been carried out in this study. The time constant $T_{D-C}$ in the BDW impedance response increased with decreasing SoC in a NiMH battery. The increase in time constant $T_{D-C}$ can be attributed to a reduction in diffusion constant $\widehat{D}_{S}$ related to reduction in the ion concentration in the electroactive material during the discharge of the battery. This study could assist the assessment of the EIS response of battery electrodes with heterogenous geometry distribution of solid particles in the electrodes. In addition, this study could assist the assessment of the state of health in battery electrodes through EIS measurements in which the concentration of the electroactive material is consumed and cannot be replace with increasing 
degradation of the battery. Other application of this study is to characterise diffusion of corrosive species characterised with BDW impedance and EIS measurements in molten salt environments for solar power plants [26].

\section{Appendix A. Newton-Raphson Iteration in Matlab® software}

\section{A1. Script to calculate the iteration process represented in Table I for FLW/} $\mathrm{Rw}=0.09$;

syms s

$\% \%$ Definition of Imaginary component through Eq. 16

$\mathrm{Zim}=\left(\operatorname{Rw} /\left((2 * \mathrm{~s})^{\wedge} 0.5\right)\right)^{*}\left(-\sinh \left((2 * \mathrm{~s})^{\wedge} 0.5\right)+\sin \left((2 * \mathrm{~s})^{\wedge} 0.5\right)\right) /\left(\cosh \left((2 * \mathrm{~s})^{\wedge} 0.5\right)+\cos \left((2 * \mathrm{~s})^{\wedge} 0.5\right)\right)$;

$\% \%$ First and second derivatives of Eq. 16

dZim=diff(Zim);

$\operatorname{ddZim}=\operatorname{diff}(\mathrm{dZim})$;

f_Zim=matlabFunction(Zim);

f_dZim=matlabFunction(dZim);

f_ddZim=matlabFunction(ddZim);

$\% \% \%$ Initial guest for SO

$\mathrm{s}=1$

$\% \%$ Iteration process Eq. 18

for $\mathrm{k}=1: 1: 100$

s1=s-(f_dZim(s)/f_ddZim(s));

$\mathrm{s}=\mathrm{s} 1$;

end

$\% \% \%$ Converged value for $s$

$\mathrm{sk}=\mathrm{s}$;

\section{A2. Script to calculate the iteration process represented in Table III for BDW}

$\mathrm{Rw}=0.4$;

$\mathrm{p}=0.78$;

$\mathrm{n}=0.5^{*} \mathrm{p}$;

syms $\mathrm{S}$

alpha $=\left(s^{\wedge} n\right) * \cos \left(n^{*}\right.$ pi/2);

beta $=\left(s^{\wedge} n\right) * \sin (n * p i / 2)$; 


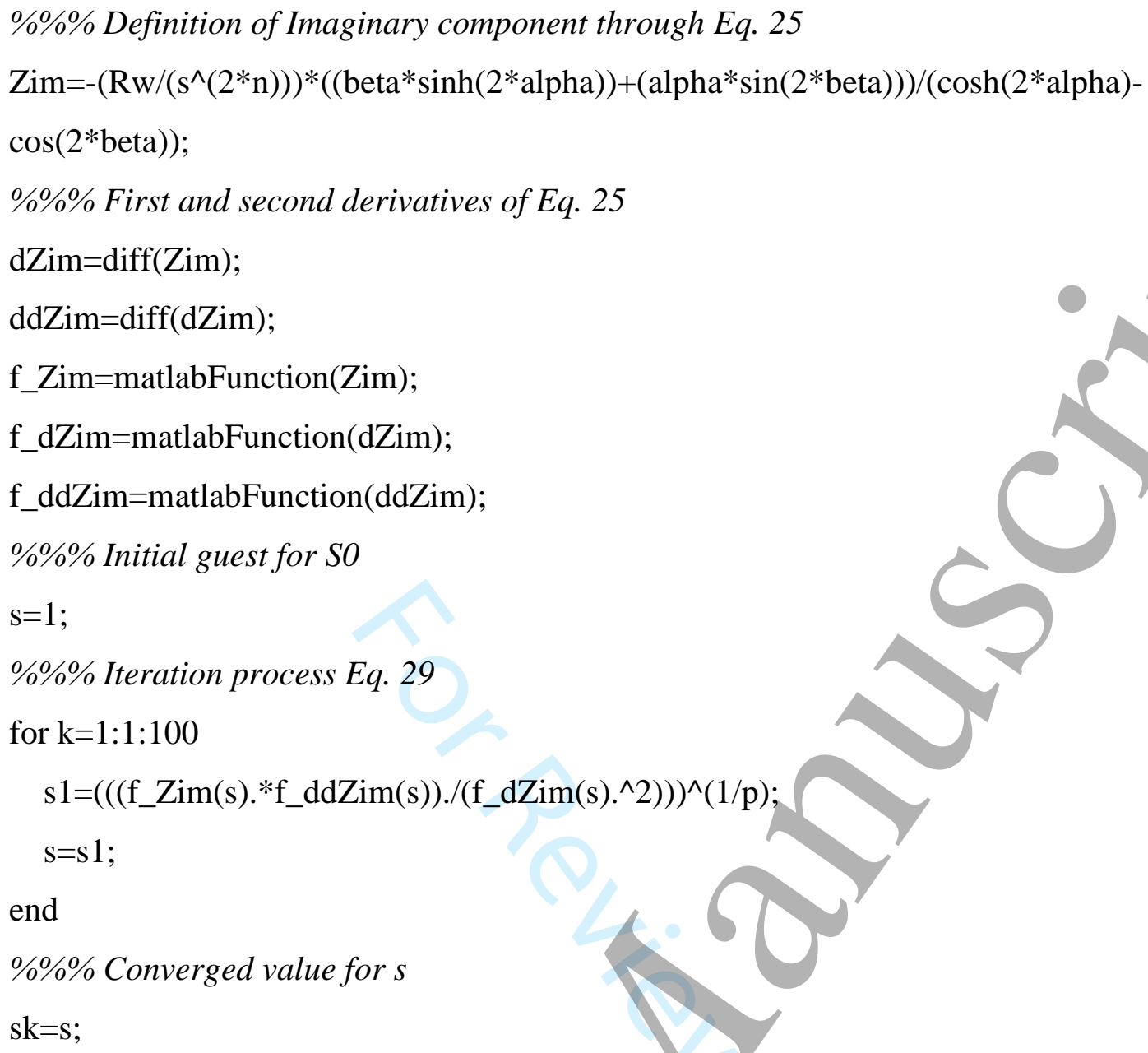

\section{List of Symbols}

$c_{O}^{*} \quad$ concentration of charge carriers in equilibrium (bulk) $\left(\mathrm{mol} / \mathrm{cm}^{3}\right)$

$D \quad$ diffusion coefficient $\left(\mathrm{m}^{2} / \mathrm{s}\right)$

$D_{S} \quad$ diffusion coefficient in solid-phase $\left(\mathrm{m}^{2} / \mathrm{s}\right)$

$\widehat{D} \quad$ parameter related to frequency dispersion $\left(\mathrm{m}^{2} \mathrm{~s}^{-\mathrm{p}}\right)$

$F \quad$ faraday constant $(96485 \mathrm{C} / \mathrm{mol})$

$f_{D-C} \quad$ frequency for diffusion-capacitive transition process $(\mathrm{Hz})$

$f_{W} \quad$ characteristic frequency $(\mathrm{Hz})$

$i \quad$ imaginary component in impedance

$p \quad$ frequency dispersion factor (dimensionless)

$R$ ideal gas constant $(8.3143 \mathrm{~J} / \mathrm{mol}-\mathrm{K})$

$R_{W} \quad$ resistance for the diffusion process $(\Omega)$

$s_{k} \quad$ converged value in Newton-iteration process (dimensionless) 
$T \quad$ temperature $(\mathrm{K})$

$T_{F L W} \quad$ characteristic time constant in FLW (s)

$T_{B D W} \quad$ characteristic time constant in BDW (s)

$T_{D-C} \quad$ time constant for the diffusion-capacitive transition process (s)

$Z_{B D W} \quad$ blocked-diffusion Warburg impedance with frequency dispersion $(\Omega)$

$Z_{F L W} \quad$ finite-length Warburg impedance $(\Omega)$

$\mathrm{z} \quad$ electrons released or consumed

$\mathrm{Z}_{\text {BDW_Im }}$ imaginary component in BDW impedance $(\Omega)$

$\mathrm{Z}_{\mathrm{FLW} \_ \text {Im }}$ imaginary component in FLW impedance $(\Omega)$

\section{Greek}

$\delta \quad$ diffusive distance in electrode (m)

$\delta_{S} \quad$ diffusive distance in solid-phase (m)

$\omega \quad$ angular frequency $(\mathrm{rad} / \mathrm{s})$

\section{References}

[1] M. Thele, M. Radin-Macukat, D. U. Sauer, O. Bohlen, and D. Linzen, in The 22nd International Battery, Hybrid and Fuel Cell Electric Vehicle Symposium \& Exposition, p. 1364, Yokohama, Japan (2006).

[2] S. Buller, M. Thele, E. Karden, and R. W. De Doncker, J. Power Sources, 113, 422 (2003).

[3] E. Kuhn, C. Forgez, P. Lagonotte, and G. Friedrich, J. Power Sources, 158, 1490 (2006).

[4] M. D. Levi, D. Aurbach, J. Phys. Chem. B, 101, (1997), 4630-4640.

[5] J. Illig, T. Chrobak, D. Klotz, E. Ivers-Tiffée, ECS Transactions, 33, (2011), 3-15.

[6] K. Onda, M. Nakayama, K. Fukuda, K. Wakahara, and T. Araki, J. Electrochem. Soc., 153, (2006), A1012A1018

[7] D.A Howey, P.D Mitcheson , V. Yufit, G. J. Offer, N. P. Brandon, IEEE Transactions on Vehicular Technology, 63, (2014), 2557-2566.

[8] A. Barai, K. Uddin, M. Dubarry, L. Somerville, A. McGordon, P. Jennings, I. Bloom, Progress in Energy and Combustion Science 72, (2019), 1-31.

[9] F. Sholz, Electroanalytical Methods, Springer, Heidelberg (2010).

[10] D. Malevich, E. Halliop, B.A. Peppley, J.G. Pharoah, K. Karan, J. Electrochem. Soc. 156 (2009) B216-B224.

[11] N. Fouquet, C. Doulet, C. Nouillant, G. Dauphin-Tanguy, B. Ould-Bouamama, J. Power Sources 159 (2006) 905-913.

[12] B. A. Boukamp, Electrochimica Acta, 252, (2017), 154-163.

[13] J.-S. Kim and S. Pyun, J. Solid State Electrochem., 15, 2447 (2011).

[14] J. Bisquert, G. Garcia-Belmonte, P. Bueno, E. Longo, and L. O. S. Bulhes, J. Electroanal. Chem., 452, (1998), 229-234

[15] J. Song and M. Z. Bazant, J. Electrochem. Soc., 160, (2013), A15-A24.

[16] J. Bisquert, and A. Compte, J. Electroanal. Chem., 499, (2001), 112-120.

[17] J. Bisquert, Electrochim. Acta, 47, (2002), 2435-2449

[18] S. Cruz-Manzo, P. Greenwood, J. Electrochem. Soc., 166, (2019), A1176-A1184.

[19] G. Barbero, Phys. Chem. Chem. Phys., 19, (2017), 32575-32579.

[20] T. Q. Nguyen and C. Breitkopf, J. Electrochem. Soc., 165, (2018), E826-E831. 
[21] T. M. W. J. Bandara and B.-E. Mellander, "Evaluation of mobility, diffusion coefficient and density of charge carriers in ionic liquids and novel electrolytes based on a new model for dielectric response," in Ionic Liquids: Theory, Properties, New Approaches, InTech, (2011).

[22] W. Peng, C. Aranda, O. M. Bakr, G. Garcia-Belmonte, J. Bisquert, ACS Energy Lett, 3, (2018), 1477-1481. [23] M. Oldenburger, B. Bedürftig, A. Gruhle, F. Grimsmann, E. Richter, R. Findeisen, J. Energy Storage, 21, (2019), 272-280.

[24] T. V. Reshetenko, G. Bender, K. Bethune, R. Rocheleau, Electrochim. Acta, 80, (2012), 368-376

[25] S. Erol, M. E. Orazem, J. Power Sources, 293, (2015), 57-64.

[26] V. Encinas-Sánchez, M. T. de Miguel, M. I. Lasanta, G. García-Martín, and F. J. Pérez, Sol. Energy Mater. Sol. Cells, 191, 157 (2019).

[27] K. Ogata, Modern Control Engineering, Fourth Edition, Pearson, (2005).

[28] J. R. Macdonald, Impedance Spectroscopy, p. 88, John Wiley \& Sons, Inc., New York (1987).

[29] N. Dimov, K. Fukuda, T. Umeno, S. Kugino, and M. Yoshio, Journal of Power Sources, 114 (2003), 88-95.

[30] S. Primdahl and M. Mogensen, J. Electrochem. Soc. 146, (1999), 2827-2833

[31] T.-Q. Nguyen, M. Glorious, and C. Breitkopf, Hindawi Advances in Mathematical Physics, (2018), 5462659, 11 pages

[32] S. Cruz-Manzo, P. Greenwood, J. Electroanal. Chem, 871, (2020), 114305.

[33] D. Gruet, B. Delobel, D. Sicsic, I. T. Lucas, V. Vivier, Electrochem. Acta, 295, (2019), 787-800.

[34] K. A. Smith, C. D. Rahn, C-Y. Wang, Energy Conversion and Management, 48, (2007), 2565-2578.

[35] K. A. Smith, C-Y. Wang, J. Power Sources, 161, (2006), 628-639.

[36] S. Cruz-Manzo, P. Greenwood, R. Chen, J. Electrochem. Soc., 164,(2017), A1446-A1453.

[37] D. Deng, Energy Science and Engineering, 3, (2015), 385-418.

[38] C. Pastor-Fernandez, K. Uddin, G. H. Chouchelamane, W. D. Widanage, J. Marco, J. Power Sources, 360, (2017), 301-318.

[39] M. Raju, M.V. Ananth, L. Vijayaraghavan, Int. J. Hydrogen Energy, 34, (2009), 3500-3505.

[40] B. Pierozynski, Int. J. Electrochem. Sci., 6, 860 (2011).

[41] J. R. Macdonald and D. R. Franceschetti, Journal of Electroanalytical Chemistry and Interfacial Electrochemistry, 99, 283 (1979).

[42] T. Jacobsen, K. West, Electrochim. Acta, 40, (1995), 255-262.

[43] M. Martínez, E. Teliz, C. F. Zinola, V. Díaz, J. Energy Storage, 27, (2020), 101067.

[44] D. Li, Y. Sun, Z. Yang, L. Gu, Y. Chen, H. Zhou, Joule, 2, (2018), 1265-1277.

[45] G. Zheng, B. N. Popov, R. E. White, J. Electrochem. Soc., 143, (1996), 834-839.

[46] T. M. W. J. Bandara, L. Ajith DeSilva, L. B. E. Gunasekara, Sunil Dehipawala, B.-E. Mellander, J. Solid State Electrochem., 24, (2020), 1207-1216.

[47] J. P. Meyers, M. Doyle, R. M. Darling, J. Newman, J. Electrochem. Soc., 147, (2000), 2930-2940

[48] G. Kobayashi, S. Nishimura, M. Park, R. Kanno, M. Yashima, T. Ida, A. Yamada, Adv. Funct. Mater., 19 (2009), 395-403.

[49] W. Waag, S. Käbitz, D. U. Sauer, Applied Energy, 102, (2013), 885-897.

[50] R. Mingant, J. Bernard, V. S. Moynot, A. Delaille, S. Mailley, ECS Trans., 33, (2011), 41.

[51] I. A. J. Gordon, S.Grugeon, H.Takenouti, B.Tribollet, M. Armand, C. Davoisne, A. Débart, S. Laruelle, Electrochim. Acta, 223, (2017), 63-73.

[52] J. Jiang, Z. Lin, Q. Ju, Z. Ma, C. Zheng, Z. Wang, Energy Procedia ,105, (2017), 844-849.

[53] O. Capron, R. Gopalakrishnan, J. Jaguemont, P. V. D. Bossche, N. Omar, J. V. Mierlo, Materials, 11, (2018), 176.

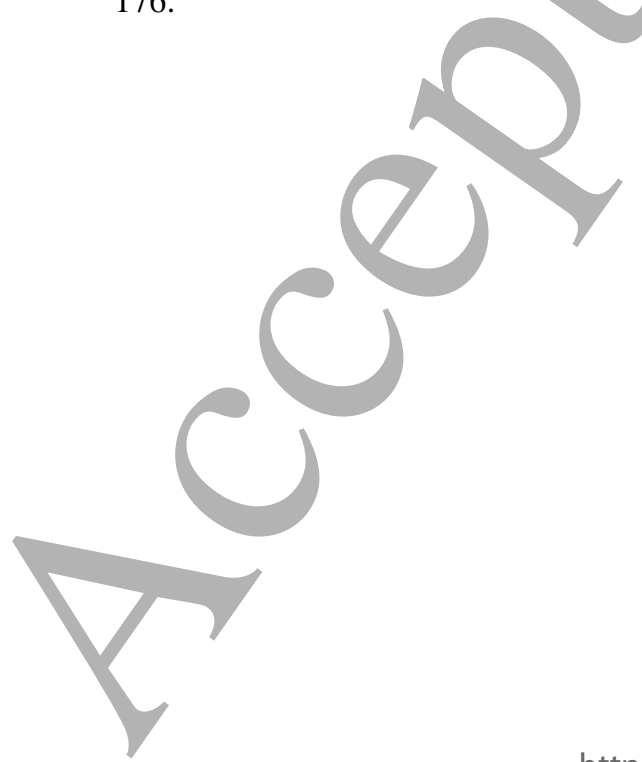

